\title{
Quantum field theory of non-abelian strings and vortices
}

\author{
Mark G. Alford \\ Institute for Theoretical Physics, University of California, Santa Barbara, CA 93106, USA
}

Kai-Ming Lee

California Institute of Technology, Pasadena, CA 91125, USA

John March-Russell

Department of Physics, Princeton University, Princeton, NJ 08540, USA

John Preskill

California Institute of Technology, Pasadena, CA 91125, USA

Received 6 January 1992

Accepted for publication 15 May 1992

\begin{abstract}
We develop an operator formalism for investigating the properties of non-abelian cosmic strings (and vortices) in quantum field theory. Operators are constructed that introduce classical string sources and that create dynamical string loops. The operator construction in lattice gauge theory is explicitly described, and correlation functions are computed in the strong-coupling and weak-coupling limits. These correlation functions are used to study the long-range interactions of non-abelian strings, taking account of charge-screening effects due to virtual particles. Among the phenomena investigated are the Aharonov-Bohm interactions of strings with charged particles, holonomy interactions between string loops, string entanglement, the transfer of "Cheshire charge" to a string loop, and domain-wall decay via spontaneous string nucleation. We also analyze the Aharonov-Bohm interactions of magnetic monopoles with electric flux tubes in a confining gauge theory. We propose that the Aharonov-Bohm effect can be invoked to distinguish among various phases of a non-abelian gauge theory coupled to matter.
\end{abstract}

\section{Introduction}

In many cases, a gauge theory that undergoes the Higgs mechanism will contain topologically stable defects. Among the defects that can occur are line defects ("cosmic strings") in $3+1$ dimensions, and pointlike vortices in $2+1$ dimensions [1]. 
If the symmetry breaking pattern and the matter content of the theory are suitable, the strings or vortices may have long-range interactions with various particles. These interactions are a consequence of the Aharonov-Bohm phenomenon - the wave function of a particle acquires a non-trivial phase when the particle is covariantly transported around the string [2,3].

This simple observation has interesting implications [4-6]. Because the Aharonov-Bohm interaction has infinite range, and no local operator can destroy an object that has an infinite-range interaction with another object, gauge theories with such interactions always respect non-trivial superselection rules. The structure of the superselection sectors can be invoked to distinguish among the various possible phases of a gauge theory. Furthermore, the existence of infinite range interactions that are fundamentally quantum mechanical exposes the limitations of the "no-hair" theorems of black-hole physics. Though a black hole may have no classical hair, it can carry quantum numbers that are detectable by means of the interaction between the black hole and a cosmic string [4,7-9].

The "Aharonov-Bohm phase" acquired by an object that circumnavigates a string is, in general, a gauge transformation contained in the unbroken gauge group. When the manifest gauge symmetry is non-abelian, this gauge transformation might not lie in the center of the group. In that case, we say that the string is "non-abelian" [10]. The physical properties of a non-abelian string are qualitatively different from the properties of an abelian string [5,6,11-13]. In particular, a loop of non-abelian string (or a pair of non-abelian vortices, in $2+1$ dimensions) can carry a non-trivial gauge charge, so that the loop has an Aharonov-Bohm interaction with other strings. Moreover, non-abelian Aharonov-Bohm interactions involve transfer of charge between string loops and charged particles. Remarkably, the charge carried by a loop (which has a topological origin) can not be localized anywhere on the string loop or in its vicinity. Following ref. [13] we will refer to such unlocalized charge as "Cheshire charge".

If we are to appeal to the Aharonov-Bohm effect to probe the phase structure of a gauge theory, or to investigate the quantum physics of black holes, we must be able to discuss interference phenomena in a language that does not rely on weak-coupling perturbative methods; we need a framework that (at least in principle) takes full account of the effects of virtual pairs and of the fluctuations of quantum fields. Such a framework was erected, for abelian strings (or vortices), in ref. [5]. There, operators were constructed that create a loop of string, or that introduce (as a classical source) the closed world-sheet of a string. The correlation functions of these operators can be studied to investigate the properties of the strings, and their Aharonov-Bohm interactions in particular.

Our main objective in this paper is to generalize the work of ref. [5] to the case of non-abelian strings. Because of the subtle and elusive physics of non-abelian strings, this generalization is not entirely straightforward [14]. Our primary motivation comes from two considerations. First, we seek assurance that the exotic 
physics of the non-abelian Aharonov-Bohm effect, previously inferred in the weak-coupling limit, actually survives in a fully quantum field-theoretic treatment. Second, we hope to construct (non-local) order parameters that can be used to classify the phases of a gauge theory.

Let us formulate the order-parameter problem more explicitly, and in so doing, review some of the principal results of ref. [5].

A gauge theory can have an interesting phase diagram. Depending on its HIggs structure and on the parameters of the Higgs potential, the theory may be in a Coulomb (massless) phase, a Higgs phase, or a confinement phase. A Higgs phase is, roughly speaking, characterized by the existence of stable magnetic flux tubes, and a confinement phase by the existence of stable electric flux tubes.

Non-local gauge-invariant order parameters can be devised that distinguish among the various phases. The expectation value of the Wilson loop operator [15] exhibits area-law behavior if there are stable electric flux tubes, and perimeter-law behavior otherwise. The expectation value of the 't Hooft loop operator [16] exhibits area-law behavior if there are stable magnetic flux tubes, and perimeter-law behavior otherwise.

These order parameters are not sufficient, however, to distinguish among all possible phases of a general gauge theory. Consider, for example, the case of an SU $(N)$ gauge theory with matter in the fundamental representation. In this theory, the Wilson loop always obeys the perimeter law, because an electric flux tube can break via nucleation of a pair. An 't Hooft loop operator can also be defined, but always obeys the area law ${ }^{\star}$. Yet the theory can have a non-trivial phase diagram. Using adjoint Higgs fields, it is possible to break the gauge group down to its center $\mathbb{Z}_{N}$. This phase, which admits free $\mathbb{Z}_{N}$ charges, is distinguishable from the confinement phase.

In ref. [5] an order parameter was described that can distinguish the free-charge phase from the confinement phase in an $\operatorname{SU}(N)$ gauge theory ${ }^{\star \star}$. The idea is that the free-charge phase supports stable magnetic flux tubes (cosmic strings), and these strings have an infinite-range Aharonov-Bohm interaction with $\mathbb{Z}_{N}$ charges. No such interaction can exist if $\mathbb{Z}_{N}$ charges are confined, or if $\mathbb{Z}_{N}$ is spontaneously broken by a Higgs field that transforms as the fundamental representation. (Indeed, the confinement phase and the "Higgs" phase with $\mathbb{Z}_{N}$ completely broken are not distinguishable [19]; this is an instance of "complementarity".)

To construct the order parameter, we must first devise an operator $F(\Sigma)$ that introduces a cosmic string world-sheet on the closed two-dimensional surface $\Sigma$.

* This is actually an oversimplification of the status of the 't Hooft loop, as we will discuss in sect. 5,7 and 9.

** This order parameter was actually discussed earlier by Fredenhagen and Marcu in ref. [17]. See also ref. [18]. 
(As we will discuss later, this operator is closely related to the 't Hooft loop-operator.) Then we consider the quantity

$$
A(\Sigma, C)=\frac{F(\Sigma) W(C)}{\langle F(\Sigma)\rangle\langle W(C)\rangle},
$$

where $W(C)$ is the (fundamental representation) Wilson loop. In the free-charge phase, we have

$$
\lim \langle A(\Sigma, C)\rangle=\exp \left(\frac{2 \pi i}{N} k(\Sigma, C)\right)
$$

Here the limit is taken with $\Sigma$ and $C$ increasing to infinite size, and with the closest approach of $\Sigma$ to $C$ also approaching infinity; $k(\Sigma, C)$ denotes the linking number of the surface $\Sigma$ and the loop $C$. On the other hand, if there are no free $\mathbb{Z}_{N}$ charges, we have

$$
\lim \langle A(\Sigma, C)\rangle=1 \text {. }
$$

The non-analytic behavior of $A(\Sigma, C)$ guarantees that a well-defined phase boundary separates the two phases. We will refer to $A(\Sigma, C)$ as the "ABOP", or "Aharonov-Bohm Order Parameter".

One of our objectives in this paper is to generalize the above construction, and to further explore its consequences. In general, it is not sufficient to determine the realization of the center of the gauge group, in order to distinguish all possible phases of a gauge theory. For example, $\mathrm{SU}(N)$ might break to a discrete subgroup $\mathrm{H}$ that is not contained in the center. Different unbroken groups (including non-abelian ones) can be distinguished according to the varieties of cosmic stings that exist, and the nature of the Aharonov-Bohm interactions of these strings with free charges. To probe the phase structure more thoroughly, we need to construct a more general $F(\Sigma)$ operator. And we will need to consider carefully the interpretation of the behavior of the corresponding $A(\Sigma, C)$ operator. The interpretation involves subtleties associated with the non-abelian Aharonov-Bohm effect.

In the example described above, there are other order parameters that can serve to distinguish the free-charge phase from the confinement phase $[17,18,20]$. But we believe that the properties of strings and of their Aharonov-Bohm interactions provide a more powerful method for classifying phases in more general cases.

The remainder of this paper is organized as follows: Sect. 2 reviews the formalism for describing configurations of many non-abelian vortices or strings. We emphasize the need to select an (arbitrary) basepoint for the purpose of defining the "magnetic flux" of a vortex, discuss the holonomy interactions between vortices, explain the origin of Cheshire charge, and note that non-abelian strings generically become entangled when they cross each other. 
In sect. 3, we discuss the properties of domain walls that are bounded by loops of non-abelian string, and observe that physically distinct strings can bound physically identical walls. Hence when a wall "decays" by nucleating a loop of string, several competing "decay channels" may be available.

Sect. 4 concerns Aharonov-Bohm interactions in abelian gauge-Higgs systems. We explore the screening of such interactions due to Higgs condensation. The observations in sect. 3 concerning domain wall decay are invoked, in order to interpret the results.

In sect. 5, we begin our analysis of the effects of quantum fluctuations on the non-abelian Aharonov-Bohm effect. We find that non-perturbative fluctuations cause ambiguities in the Aharonov-Bohm "phase" beyond those that occur in leading semiclassical theory. We construct operators that create configurations of many non-abelian strings. Two types of operators are considered. One type introduces classical string sources on closed world-sheets. The other type creates (or destroys) dynamical string loops.

A subtle aspect of the construction is that, for both types, the many string configurations are coherent. This means the following: The "magnetic flux" carried by a string can be characterized by an element $a$ of the unbroken gauge group $\mathrm{H}$. If an object that transforms as the representation $(\nu)$ of $\mathrm{H}$ is transported about this string, the Aharonov-Bohm phase that it acquires, averaged over a basis for the representation, is

$$
\frac{1}{n_{\nu}} \chi^{(\nu)}(a)
$$

where $n_{\nu}$ is the dimension of $(\nu)$ and $\chi^{(\nu)}$ is its character [6,21]. Now if an $a$ string and a $b$ string are combined incoherently, the averaged phase acquired by an object that traverses the two strings in succession is

$$
\frac{1}{n_{\nu}} \chi^{(\nu)}(a) \frac{1}{n_{\nu}} \chi^{(\nu)}(b)
$$

that is, it is the product of the Aharonov-Bohm factors associated with the two individual strings. But if the two strings are patched together coherently, the averaged phase becomes

$$
\frac{1}{n_{\nu}} \chi^{(\nu)}(a b)
$$

This property, that the Aharonov-Bohm factor associated with a pair of coherently combined strings is not just the product of the Aharonov-Bohm factors of the two individual strings, is a distinguishing feature of the non-abelian Aharonov-Bohm effect, and one of the goals of our work has been to better understand how this 
coherence is maintained when quantum fluctuations of fields are fully taken into account.

Correlation functions of the operators constructed in sect. 5 are used in sect. 6 to investigate Aharonov-Bohm interactions in a pure gauge theory, at both strong and weak coupling. In sect. 7 , we use these operators to study the quantum-mechanical mixing between different string states first discussed in ref. [21].

Holonomy interactions between pairs of vortices, or pairs of string loops, are considered in sect. 8. Correlation functions are used to demonstrate holonomy scattering and string entanglement. We also analyze a correlation function that realizes the transfer of charge from a charged source to a loop of string, and the subsequent detection of the (Cheshire) charge on the loop by means of its Aharonov-Bohm interaction with yet another loop of string.

In sect. 9, we consider non-abelian gauge-Higgs systems; we analyze the consequences of the Higgs mechanism for the stability of strings, and for the screening of Aharonov-Bohm interactions. We discuss how non-local order-parameters can be used to explore the phase structure of such a system.

Sect. 10 concerns the properties of dynamical magnetic monopoles, in a confining gauge theory. We construct generalizations of the Wilson and 't Hooft operators for a theory with dynamical monopoles, and use these operators to demonstrate the Aharonov-Bohm interaction between monopoles and electric flux tubes [22,23].

Appendix A provides some additional details concerning some of the lattice calculations that are mentioned in the text of the paper.

\section{Non-abelian vortices and strings}

We will briefly review the formalism [24] for describing configurations of many vortices (in two spatial dimensions) or many strings (in three spatial dimensions). Our purpose is to remind the reader of two peculiar features of non-abelian strings. First, there is an ambiguity when two or more loops of string are patched together to construct a multi-string configuration. Second, a loop of string can carry charges, and can exchange charge with other objects by means of the non-abelian Aharonov-Bohm effect. An understanding of these features is needed in order to interpret the behavior of the correlation functions that we will construct.

\subsection{MANY-VORTEX CONFIGURATION}

We will consider vortices first, and discuss strings later. A single isolated vortex can be associated with an element of the unbroken gauge group $\mathrm{H}$, according to

$$
a\left(C, x_{0}\right)=P \exp \left(i \int_{C, x_{0}} A \cdot \mathrm{d} x\right) \text {. }
$$


Here $C$ is a closed oriented path, far from the vortex core, that encloses the vortex and begins and ends at the point $x_{0}$. This group element $a\left(C, x_{0}\right)$ is invariant under deformations of the path $C$ that keep $x_{0}$ fixed and that avoid the vortex core. An object that transforms as the irreducible representation $(\nu)$ of $\mathrm{H}$ acquires the "Aharonov-Bohm" phase $D^{(\nu)}\left(a\left(C, x_{0}\right)\right)$ when covariantly transported around the vortex. We require $a \in \mathrm{H}$, because the Higgs fields that drive the symmetry breakdown must return to their original values when so transported. The vortices can be topologically classified with the topological charge taking values in $\pi_{0}(\mathrm{H})$; that is, a vortex with "flux" $a \in \mathrm{H}$ can be smoothly deformed to another vortex with flux $b \in \mathrm{H}$ if and only if $a$ and $b$ lie in the same connected component of $\mathrm{H}^{*}$. If $\mathbf{H}$ is a discrete group (as we will usually assume in this paper) the topological charge is specified by an element of $\mathrm{H}$.

Similarly, we may associate $n$ elements of $\mathrm{H}$ with a configuration of $n$ vortices. To do so, we must choose $n$ standard paths, all beginning and ending at the same point $x_{0}$, that circumnavigate the various vortices [24].

This description of the $n$-vortex configuration evidently suffers from some ambiguities. First, it is not gauge invariant. Under a gauge transformation that takes the value $h \in \mathrm{H}$ at the point $x_{0}$ (and so preserves the Higgs order parameter at that point), the elements $a_{1}, a_{2}, \ldots, a_{n}$ transform according to

$$
a_{i} \rightarrow h a_{i} h^{-1} .
$$

Thus, the configurations of a single vortex are labeled by the conjugacy classes of $\mathrm{H}$. But the gauge freedom for many vortices involves just one overall conjugation. This means that specifying the positions and classes of all vortices is not sufficient to characterize the multi-vortex configuration. Single vortices can be patched together in various ways to construct different multi-vortex states.

Another ambiguity concerns the choice of the paths $C_{i}$ that enclose the vortices. The paths that begin and end at $x_{0}$ and avoid the cores of $n$ vortices fall into homotopy classes; these classes are the elements of $\pi_{1}\left(\mathrm{M}_{n}, x_{0}\right)$, the fundamental group of $\mathrm{M}_{n}$, the plane with $n$ punctures. (This group is $\mathrm{F}_{n}$, the free group with $n$ generators.) By assigning elements of $\mathrm{H}$ to each of the generators of $\pi_{1}\left(\mathrm{M}_{n}, x_{0}\right)$, we have defined a homomorphism from $\pi_{1}\left(\mathrm{M}_{n}, x_{0}\right)$ into $\mathrm{H}$. This homomorphism (modulo one overall conjugation) characterizes the configuration of $n$ vortices.

But this homomorphism is still ambiguous, because the $n$ generators can be chosen in various ways. Consider in particular the case of two vortices, as shown in fig. 1. Standard paths $\alpha_{1}$ and $\alpha_{2}$ have been chosen in fig. 1a that wind counterclockwise around the two vortices. A topologically distinct choice for the path around vortex 1 is shown in fig. 1c, and a distinct choice for the path around vortex

* Here, we have implicitly defined $\mathrm{H}$ as the unbroken subgroup of a simply connected underlying (spontaneously broken) gauge group. The global topology of the underlying group is irrelevant, however, for the purpose of classifying the Aharonov-Bohm interactions of the vortices. 


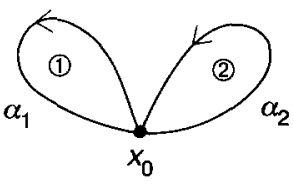

(a)

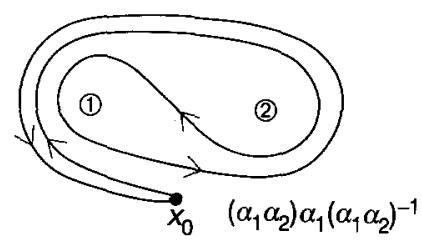

(c)

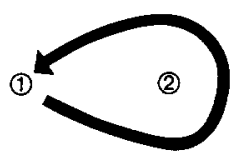

(b)

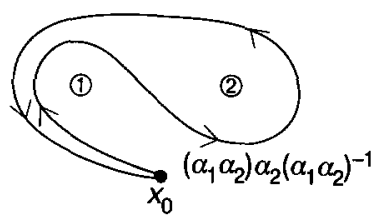

(d)

Fig. 1. (a) Standard paths $\alpha_{1}$ and $\alpha_{2}$, based at $x_{0}$, that enclose vortex 1 and vortex 2. (b) Vortex 1 winds around vortex 2. (c) Path that, during the winding of vortex 1 around vortex 2 , gets dragged to $\alpha_{1}$. (d) Path that gets dragged to $\alpha_{2}$ during the winding.

2 is shown in fig. 1d. Suppose now that vortex 1 winds around vortex 2 (in the sense defined by the path $\alpha_{2}$ ), and returns to its original position, as in fig. 1b. We may deform our paths during the winding so that no vortex ever crosses any path; then each path is mapped to the same group element after the winding as before the winding is the same as the group element associated with $\alpha_{1}$ after the winding. But after the winding, the final deformed path is not homotopically equivalent to the initial path. Therefore, the homomorphism that maps $\pi_{1}\left(\mathrm{M}_{2}, x_{0}\right)$ to the group has changed.

Suppose, for example, that initially $\alpha_{1}$ is mapped to $a_{1}$ and $\alpha_{2}$ is mapped to $a_{2}$. We wish to determine the final values, after the winding, of the group elements associated with the paths $\alpha_{1}$ and $\alpha_{2}$. For this purpose, it is convenient to notice that, during the winding, the path shown in fig. 1c is "dragged" to $\alpha_{1}$. Therefore, the group element associated with this path before the winding is the same as the group element associated with $\alpha_{1}$ after the winding. We see that this path can be expressed as $\left(\alpha_{1} \alpha_{2}\right) \alpha_{1}\left(\alpha_{1} \alpha_{2}\right)^{-1}$, where $\alpha_{1} \alpha_{2}$ denotes the path that is obtained by traversing $\alpha_{2}$ first and $\alpha_{1}$ second. The initial homomophism maps this path to a path-ordered exponential with the value

$$
a_{1}^{\prime}=\left(a_{1} a_{2}\right) a_{1}\left(a_{1} a_{2}\right)^{-1} \text {. }
$$

Similarly we note that the path shown in fig. $1 \mathrm{~d}$ is dragged during the winding to $\alpha_{2}$. This path is $\left(\alpha_{1} \alpha_{2}\right) \alpha_{2}\left(\alpha_{1} \alpha_{2}\right)^{-1}$, and is mapped by the initial homomorphism to

$$
a_{2}^{\prime}=\left(a_{1} a_{2}\right) a_{2}\left(a_{1} a_{2}\right)^{-1} \text {. }
$$

We conclude that the final homomorphism after the winding maps $\alpha_{1}$ to $a_{1}^{\prime}$ and maps $\alpha_{2}$ to $a_{2}^{\prime}$. Winding vortex 1 around vortex 2 has changed the two-vortex state 
(if $a_{1}$ and $a_{2}$ do not commute) - both group elements have become conjugated by $a_{1} a_{2}$.

This change in the two-vortex state is not a mere mathematical curiosity. The physical interpretation is that there is a long-range interaction between non-commuting vortices $[24,25]$. We refer to this as the "holonomy interaction." It is a type of Aharonov-Bohm interaction, but we prefer not to use that name in this context. When the number of vortices is three or more, these holonomy interactions can be quite complicated.

Finally, another source of ambiguity concerns the choice of the basepoint $x_{0}$. Winding the basepoint around some of the vortices (or some of the vortices around the basepoint) also changes our description of the multi-vortex configuration. This feature is not of much physical interest. When it is convenient, we will consider the basepoint to be far from all vortices, so that winding of vortices around the basepoint need not be considered.

The gauge-invariant content of the classical $n$-vortex configuration can be encoded in the Wilson loop operators

$$
W^{(\nu)}(C)=\frac{1}{n_{\nu}} \chi^{(\nu)}\left(P \exp \left(i \oint_{C} A \cdot \mathrm{d} x\right)\right),
$$

where $\chi^{(\nu)}$ denotes the character and $n_{\nu}$ the dimension of irreducible representation $(\nu)$. If $C$ is a closed path around a single vortex, then $W^{(\nu)}(C)$, for all $(\nu)$, provides sufficient information to identify the class to which the vortex belongs. Likewise, $W^{(\nu)}(C)$, for all $(\nu)$ and all $C$ in $\pi_{1}\left(\mathrm{M}_{n}\right)$ suffices to determine the $n$-vortex configuration, up to overall configuration. It is not sufficient though, to know $W^{(\nu)}\left(C_{i}\right)$ for all the generators $C_{i}$ of $\pi_{1}\left(\mathrm{M}_{n}\right)$; this determines only the class of each vortex, but not how the vortices are patched together. It is therefore more convenient and efficient to fix the gauge at the basepoint and assign group elements to standard paths around the vortices than to give a fully gauge-invariant description in terms of Wilson loops.

When the unbroken gauge group $\mathrm{H}$ is discrete, one sometimes says that the theory respects a "local discrete symmetry" [4-6,26-29] *. This terminology is used because a field that is covariantly constant outside the vortex core is typically not single valued - on a closed path that encloses a vortex, a covariantly constant field is periodic only up to the action of an element of $\mathrm{H}$. (And, conversely, a field that is periodic cannot be covariantly constant.) But the phrase "local discrete symmetry" should not be misunderstood. Often, when we say that a symmetry is local, we mean that all physical states are required to be invariant under the symmetry. That

* The first formulation of local discrete symmetries in the continuum was given by Kiskis [26] although not in the context of spontaneously broken gauge theories. 
is not what is meant here. Isolated $\mathrm{H}$-charges can exist, and can, in fact, be detected at infinite range via their Aharonov-Bohm interactions with vortices.

\subsection{CHESHIRE CHARGE}

Aside from their topological charges, vortices can also carry ordinary $\mathrm{H}$ quantum numbers $[5,12,13]$. These are easiest to discuss if we consider a vortex-antivortex pair, with trivial total topological charge. In this configuration, the vortex is described by a group element $a$, and the antivortex by $a^{-1}$. But under H-transformations, $a$ mixes with other representatives of the conjugacy class to which it belongs.

The action of $\mathbf{H}$ on the members of a class $\alpha$ defines a (reducible) representation which we denote as $D^{(\alpha)}$. In $D^{(\alpha)}$, each element of $\mathrm{H}$ is represented by a permutation of the class, according to

$$
D^{(\alpha)}(h): \quad|a\rangle \rightarrow\left|h a h^{-1}\right\rangle, \quad a \in \alpha .
$$

This representation can be decomposed into irreducible representations of $H$. The physical interpretation is that the vortex pair can carry $\mathrm{H}$-charge. This charge is a property of the vortex-antivortex composite system and is not localized on either vortex or antivortex. It can be detected by means of the Aharonov-Bohm interaction of the composite with another vortex.

For each class $\alpha$ in $\mathrm{H}$ there is a unique state that can be constructed that is uncharged (transforms trivially under $\mathrm{H}$ ); it is the superposition of group eigenstates

$$
\frac{1}{\sqrt{n_{\alpha}}} \sum_{a \in \alpha}|a\rangle
$$

(where $n_{\alpha}$ denotes the order of the class). Let us consider what happens to this state when an object that transforms as the irreducible representation $(\nu)$ of $\mathrm{H}$ passes between the two vortices.

The composite system consisting of the vortex pair and the charged projectile has a well-defined $\mathrm{H}$-charge; the composite transforms according to a representation of $\mathbf{H}$ that could be inferred by studying the Aharonov-Bohm interaction of the composite with other vortices. (It is to ensure this that we consider a pair of vortices with trivial total topological charge.) Interactions between the vortex pair and the projectile cannot change this total $\mathrm{H}$-charge. From this observation, we can infer how the charge of the vortex pair changes when a charged object winds around one of the vortices [30].

It is convenient to imagine that the vortex pair actually interacts with a 


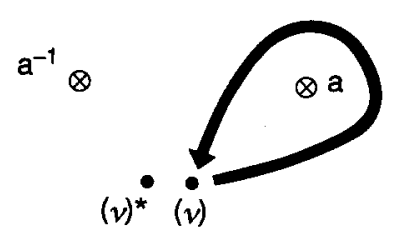

Fig. 2. A particle transforming as the representation ( $\nu$ ) of $\mathrm{H}$ interacts with a vortex pair. The particle and its antiparticle are initially prepared in an uncharged (gauge-singlet) state. After the particle winds around the vortex, the particle-antiparticle pair has acquired a non-trivial charge.

particle-antiparticle pair, combined in the trivial representation of $\mathrm{H}$, so that the total H-charge of particles plus vortices is trivial. Then, if one particle winds around one vortex as in fig. 2, the charge transferred to the vortex pair is opposite to the charge transferred to the particle pair; the vortices are in a state transforming as the representation $(\mu)^{*}$, if the particle pair transforms as $(\mu)$.

The charge-zero state of the particle-antiparticle pair has the group-theoretic structure

$$
\frac{1}{\sqrt{n_{\nu}}}\left|e_{i}^{(\nu) *} \otimes e_{i}^{(\nu)}\right\rangle
$$

(summed over $i$ ) where $\left\{e_{i}^{(\nu)}\right\}$ denotes a basis for the vector space on which the representation $(\nu)$ acts, and $n_{\nu}$ is the dimension of that representation. After the particle winds around a vortex with flux $a \in \mathrm{H}$, this state becomes

$$
\frac{1}{\sqrt{n_{\nu}}}\left|e_{i}^{(\nu) *} \otimes e_{j}^{(\nu)}\right\rangle D_{j i}^{(\nu)}(a)
$$

We may regard this state as a vector in the space on which the representation $D^{(\nu) *} \otimes D^{(\nu)}$ acts, expanded in terms of the natural basis $\left|e_{i}^{(\nu) *} \otimes e_{j}^{(\nu)}\right\rangle$. This vector can also be expanded in terms of the basis that block-diagonalizes the representation, as

$$
\frac{1}{\sqrt{n_{\nu}}} D^{(\nu)}(a)=\sum_{\mu, y} c_{\mu, y} v^{(\mu), y} .
$$

Here, $v^{(\mu), y}$ is a unit vector in the irreducible subspace acted on by the representation $(\mu), y$; the index $y$ occurs because a given irreducible representation may occur in $D^{(\nu) *} \otimes D^{(\nu)}$ more than once.

We may interpret

$$
p_{\mu}=\sum_{y}\left|c_{\mu, y}\right|^{2}
$$


as the probability that the final state of the particle pair transforms as $D^{(\mu)}$; correspondingly, it is the probability that, after interacting with the projectile, the vortex pair transforms as $D^{(\mu) *}$ (The $|c|$ 's do not depend on how the class representative $a$ is chosen.) Of course, $p_{\mu}$ can be non-vanishing only if $D^{(\mu) *}$ is one of the irreducible constituents of $D^{(\alpha)}$.

Thus we see that charge is transferred to the vortex pair, via the non-abelian Aharonov-Bohm effect, when a charged particle winds around the vortex $[5,12,13]$; an initially uncharged vortex pair becomes a non-trivial superposition of charge eigenstates. This is "Cheshire charge." The charge is a global property of the vortex pair, and is not localized on the vortices.

\subsection{STRINGS}

The above description of vortices is easily translated into appropriate language to describe loops of string. A multi-string configuration can be characterized by assigning elements of $\mathrm{H}$ to each of a set of standard paths (beginning and ending at a basepoint $x_{0}$ ) that link the string loops. Again, the paths that begin and end at $x_{0}$ and avoid the cores of all strings fall into homotopy classes; these classes are the elements of $\pi_{1}\left(\mathrm{M}, x_{0}\right)$, where $\mathrm{M}$ now denotes $\mathbb{R}^{3}$ with all string loops removed.

Thus, when many strings are present, there is an ambiguity in the choice of the "standard path" that links a given string, and a corresponding ambiguity in the assignment of group elements to strings. The physics associated with this ambiguity is that there is a long-range holonomy interaction between string loops.

In fig. 3a, standard paths $\alpha$ and $\beta$ have been introduced that wind around two string loops. Suppose that, initially the homomorphism maps $\alpha$ to the group element $a$, and maps $\beta$ to the group element $b$. Now suppose that the $b$ string loops winds through the $a$ loop, in the sense defined by $\alpha$. During the winding, the path shown in fig. $3 \mathrm{~b}$ is dragged to $\beta$. Thus, the flux associated with this path before the winding is the same as the flux associated with $\beta$ after the winding. This path can be expressed as $\alpha \beta \alpha^{-1}$, and so is mapped by the initial homomorphism

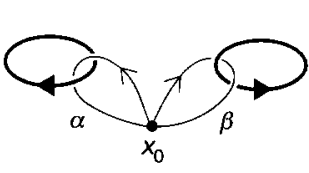

(a)

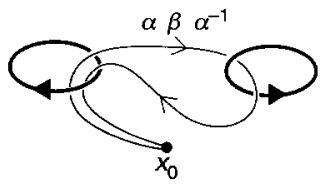

(b)

Fig. 3. (a) Standard paths $\alpha$ and $\beta$, based at ${ }_{0}$, associated with the $a$-string and the $b$-string. (b) Path that is dragged to the path $\beta$ as the loop of $b$-string winds through the loop of $a$-string (in the sense defined by $\alpha$ ). 
to the group element $a b a^{-1}$. We conclude that the final homomorphism after the winding maps $\beta$ to

$$
b^{\prime}=a b a^{-1}
$$

and maps $\alpha$ to $a^{\prime}=a$. When a loop of $b$ string winds through a loop of $a$ string, its flux becomes conjugated - it becomes an $a b a^{-1}$ string.

For future reference, we remark that fig. 3 has an alternative interpretation. The path shown in fig. $3 \mathrm{~b}$ will also be dragged to $\beta$ if the $a$ string winds around the basepoint $x_{0}$, without the $b$ loop passing through the $a$ loop at all. Of course, the basepoint $x_{0}$ is completely arbitrary, and selected by convention, so this process has no actual physical effect on the $b$ loop. Yet, the effect of parallel transport around the path $\beta$ will be different than before, after the $a$ loop has lassoed the basepoint. We will return to this point in sect. 5 .

Furthermore, a string loop, like a pair of vortices, can carry an H-charge, and can exchange charge with other charged objects by means of the Aharonov-Bohm effect.

\subsection{BRANCHING AND ENTANGLEMENT}

Another property of strings will be relevant to our subsequent analysis; namely, that strings can branch. This feature is illustrated in fig. 4. There a string labeled by $c=b a$ splits into two strings with labels $a$ and $b$. If the gauge group in non-abelian, then the precise rules for composing the group elements at a branch point depend on our conventions - these (arbitrary) conventions associate each string with a path around the string that begins and ends at the basepoint, as shown in fig. 4.

Related to the branching phenomenon is another generic feature of nonabelian cosmic strings - strings that are labeled by noncommuting group elements become entangled when they cross [31]. Fig. 5 illustrates that when an $a$-string and a $b$-string cross, they become connected by a segment of $c$-string. The mathematics underlying this entanglement is very similar to that underlying the holonomy interaction between two non-abelian vortices, in two spatial dimensions.

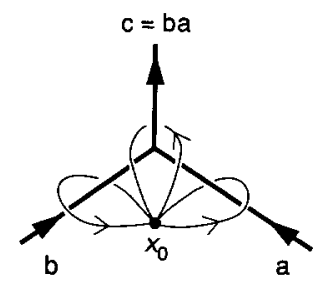

Fig. 4. The branching of a $c=b a$ string into an $a$ string and a $b$ string. The standard paths, based at $x_{0}$, associated with each string are indicated. 


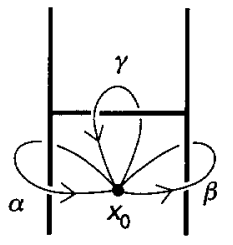

(a)

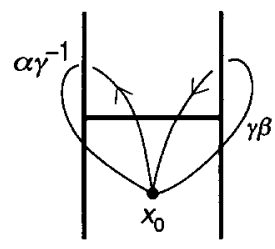

(c)

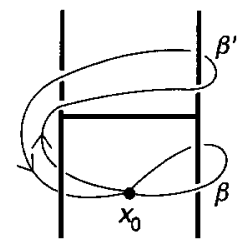

(b)

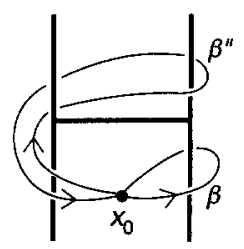

(d)

Fig. 5. (a) The standard paths $\alpha, \beta$ and $\gamma$ associated with entangled strings. These paths are mapped by the string homomorphism to group elements $a, b$ and $c$. (b) The paths $\beta$ and $\beta^{\prime}$ are both mapped to $b$, if the bridge connecting the strings can be removed by crossing the strings. (c) Standard paths associated with the "upper halves" of entangled strings. (d) The paths $\beta$ and $\beta$ " are both mapped to $b$, if the bridge can be removed by crossing the strings "the other way".

Let us calculate the flux $c$ carried by the string segment that connects the $a$-string and the $b$-string, after the crossing. First, we establish our conventions by choosing standard paths $\alpha, \beta$ and $\gamma$ that encircle the string, as in fig. 5a. The group element $c$ associated with $\gamma$ can be determined if we demand that the bridge connecting the strings can be removed by re-crossing the strings. However, even once we have fixed all our conventions, the element $c$ is not uniquely determined-there are two possible choices. This ambiguity arises because a crossing event involving two oriented strings has a handedness. A useful way to think about the handedness of the crossing is to imagine that the $a$ and $b$ strings are actually large closed loops. When the string loops cross, they become linked. But the linking number can be either +1 or -1 . (That is, we may consider a surface bounded by one of the loops. This surface inherits an orientation from the loop, defined (say) by the right-hand rule. If the other loop pierces the surface in the same sense as the outward-pointing normal, the linking number is +1 ; otherwise, -1.) The two possible linking numbers correspond to the two possible types of crossing events.

For one of the two types of crossings (the case in which the loops have linking number -1 ), the paths $\beta$ and $\beta^{\prime}$ in fig. $5 b$ must be mapped to the same group element. In terms of the standard paths defined in fig. 5a, we have $\beta^{\prime}=\alpha^{-1} \gamma \beta \alpha$. 
(Recall our notation - in a composition of paths, the path on the right is traced first.) We therefore find $b=a^{-1} c b a$, or

$$
c=a b a^{-1} b^{-1}
$$

The connection with the holonomy interaction between vortices is clarified by fig. 5c, where standard paths $\alpha \gamma^{-1}$ and $\gamma \beta$ are shown that wind around the "upper halves" of the entangled strings. Since $a c^{-1}=(a b) a(a b)^{-1}$ and $c b=(a b) b(a b)^{-1}$, the "flux" carried by the upper half strings differs from the flux carried by the lower half strings by conjugation by $a b$ (just as winding an $a$-vortex counterclockwise around a $b$-vortex causes the flux of both vortices to be conjugated by $a b$ ).

For the other type of crossing (the case in which the loops have linking number +1 ), the paths $\beta$ and $\beta^{\prime \prime}$ in fig. 5d must be mapped to the same group element. This path is $\beta^{\prime \prime}=\alpha \beta \gamma \alpha^{-1}$, so that $b=a b c a^{-1}$, or

$$
c=b^{-1} a^{-1} b a
$$

Now the upper half strings carry flux $(a b)^{-1} a(a b)$ and $(a b)^{-1} b(a b)$, which is just the change in a two-vortex state that results from winding an $a$-vortex around a $b$-vortex in the clockwise sense.

\section{Non-abelian walls bounded by strings}

If a gauge theory respects a local discrete symmetry $G$, then, as we have seen, the theory admits cosmic strings that are classified by the elements of $G$. But if the group $G$ is spontaneously broken to a subgroup $H$, then some of these strings become boundaries of domain walls [32]. Here we will consider some of the properties of domain walls bounded by strings. It is necessary to understand these properties, in order to interpret the behavior of our correlation functions, and to use them to probe the phase structure of a model.

In this section, as before, we assume that a standard choice of gauge has been made at a standard basepoint. This choice allows us to assign a definite group element to each cosmic string, and also fixes the embedding of the unbroken group $\mathrm{H}$ in $\mathrm{G}$.

\subsection{CLASSIFYING WALLS BOUNDED BY STRING}

If a discrete gauge group $G$ is spontaneously broken to a subgroup $H$, then the cosmic strings of the theory are classified by elements of $H$. The elements of $G$ that are not in $\mathrm{H}$ are associated not with isolatcu strings, but rather with strings that bound segments of domain wall. 


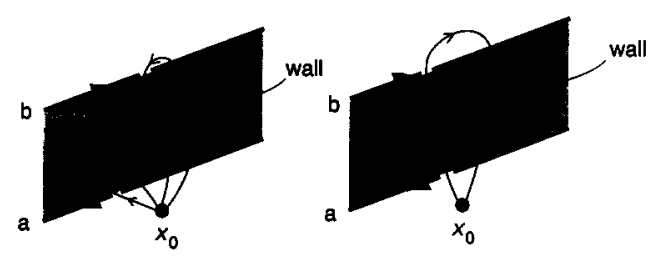

(a)

(b)

Fig. 6. (a) Standard paths that are mapped by the string homomorphism to elements $a, b \in \mathrm{G}$ that are not in the unbroken group $\mathrm{H}$. Each string is the boundary of a domain wall. (b) A path that is mapped to $b^{-1} a \in \mathrm{H}$. This path does not cross the wall.

There is not, however, a one-to-one correspondence between elements of $\mathrm{G}$ that are not in $\mathrm{H}$ and the physically distinguishable types of wall. This is because a single type of wall can be bounded by more than one type of string. As is illustrated in fig. 6, strings labeled by $a$ and $b$ can bound the same wall, if $h=b^{-1} a$ is in the unbroken group $\mathrm{H}$. The composite of the two strings is an object that does not end on a wall, which means that the wall that ends on an $a$ string must also end on a $b$-string (with conventions chosen as in the figure). Thus, no locally measurable property distinguishes a wall that ends on a $a$-string from a wall that ends on a $b$-string, if $b^{-1} a \in \mathrm{H}$. In other words, walls are classified by the cosets $\{g \mathrm{H}\}$ of $\mathrm{H}$ in $\mathrm{G}$ (with the trivial coset corresponding to the trivial wall, i.e. no wall at all). Whether the walls are labeled by left or right cosets is a matter of convention.

Another way to think about this classification is in terms of the discontinuity of the order parameter across the domain wall. Suppose that the Higgs field $\Phi$ that drives the breakdown from $\mathrm{G}$ to $\mathrm{H}$ transforms as the representation $R$ of $\mathrm{G}$, so that $\mathrm{D}^{(R)}(h) \Phi=\Phi$ for $h \in \mathrm{H}$. Across the wall labeled by $a$, the order parameter jumps from $\Phi$ to $D^{(R)}(a) \Phi$. This discontinuity is independent of the choice of coset representative.

To avoid confusion, we should remark that the classification that we have just described is not the usual classification of domain walls in a spontaneously broken gauge theory. The point is that we are not classifying the domain walls that are absolutely stable, as in the standard analysis of topological defects in gauge theories. Instead, we are classifying the different walls that can end on strings.

Notice that the criterion for two strings to be boundaries of the same wall is not the same as the criterion for two strings to be (locally) indistinguishable objects. Strings labeled by $a$ and $b$ have the same structure if $a$ and $b$ are related by $a=h b h^{-1}$ for some $h \in \mathrm{H}$. Two elements of $\mathrm{G}$ can be in the same $\mathrm{H}$-coset without being related in this way. Therefore, the same type of wall can have more than one type of boundary in general. 


\subsection{WALL DECAY}

The walls described above, those associated with elements of $G$ that are not in the unbroken group $\mathbf{H}$, are unstable. Such a wall will decay by nucleating a loop of string by quantum tunneling; the loop then expands, consuming the wall $[32,1]$.

As we have seen, it is possible for one type of wall to end on more than one type of string. Hence, the wall may be able to decay in more than one way. Furthermore, if a large segment of wall bounded by an $a$ string decays, two different decay modes that are locally indistinguishable may be globally distinguishable. For example, it may be that $b$ and $b^{\prime}$ are both in the same $\mathrm{H}$-coset as $a$, and also that $b^{\prime}=h b h^{-1}$, for some $h \in \mathrm{H}$. Then the wall can decay by nucleating either a $b$-string or a $b^{\prime}$-string, and the strings look the same locally. But $b^{-1} a$ and $b^{\prime-1} a$ need not be the same element of $H$. Then the ribbon of wall that is produced by the nucleation of the loop (see fig. 6), is different in the two cases.

If a large loop of $a$-string is prepared, which bounds a wall, then the wall can decay in any of the available channels. The string with the lowest tension is the most likely to nucleate, but all strings that can bound the wall nucleate at some non-vanishing rate. Many holes eventually appear in the wall, and the boundaries of the holes (the strings) expand due to the tension in the wall. Eventually the holes collide.

When an $a$-hole meets a $b$-hole, junctions form, and an $a b^{-1}$ string appears that bridges the hole. (See fig. $7^{\star}$.) It may be that the $b$-string has higher tension than the $a$-string. Then the junctions will get pulled around the boundary of the hole. They annihilate, liberating a $a b^{-1}$ string from the decaying wall system.

This scenario shows that, when the wall bounding a very large loop of string decays, we may regard the decay process as dominated by the strings on which the wall can end that have the smallest possible tension. This insight will help us to interpret the behavior of the order parameter $A(\Sigma, C)$. If an operator creates a world sheet of an $a$-string, which bounds a wall, then the Aharonov-Bohm interaction with a charge whose world line winds around the string world-sheet will be the same as the Aharonov-Bohm interaction of the charge with the $a b^{-1} \in \mathbf{H}$ string, if the $b$-string is the lightest one that can nucleate on the wall.

For completeness, we note another structure that can arise in walls bounded by strings. We have seen that a $b a$ string can branch into an $a$-string and a $b$-string. It may be that all three strings are boundaries of walls. Then a possible configuration is shown in fig. 8. Here the $b a$ string is a vein in the wall, where the type of wall bounded by the $a$-string joins the type of wall bounded by the $b$-string.

\footnotetext{
* Whether we identify the flux of the string that stretches across the hole in fig. $7 \mathrm{~b}$ as $a b^{-1}$ or $b^{-1} a$ depends on our conventions.
} 


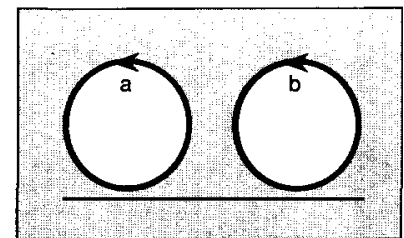

(a)

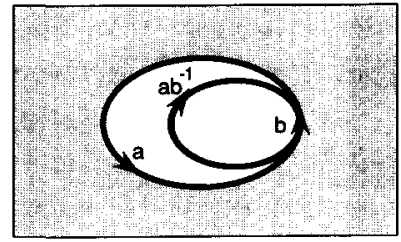

(c)

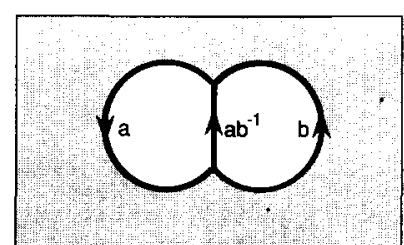

(b)

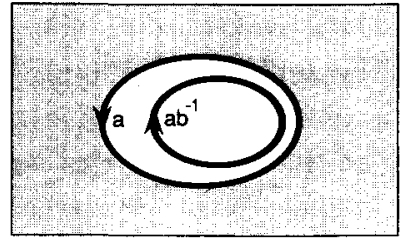

(d)

Fig. 7. (a) Two holes appear in a domain wall, due to the spontaneous nucleation of loops of $a$-string and $b$-string. (b) The holes meet and coalesce. A segment of $a b^{-1}$ string appears that bridges the hole. (c) The $b$-string has higher tension than the $a$-string, and so pulls the string junctions toward each other. (d) A loop of $a b^{-1}$ string breaks free from the decaying wall.

\section{The abelian order parameter}

The "abelian" order parameter introduced in ref. [5] can be used to probe the realization of the center of the gauge group. Here we will briefly describe how this order parameter is used to distinguish the various phases of a $\mathbb{Z}_{N}$ gauge-spin system on the lattice.

The model we consider has gauge variables $U_{l}$ residing on the links (labeled by $l$ ) of a cubic lattice, and spin variables $\phi_{i}$ residing on the sites (labeled by $i$ ). Both gauge and spin variables take values in

$$
\mathbb{Z}_{N} \equiv\{\exp (2 \pi i k / N), k=0,1,2, \ldots, N-1\},
$$

The (euclidean) action of the model is

$$
S=S_{\text {gauge }}+S_{\text {spin }},
$$

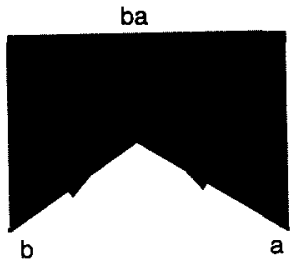

Fig. 8. A vein in a wall. 


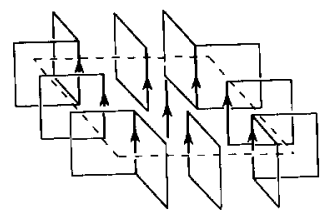

Fig. 9. The closed curve $\Sigma$ in $2+1$ dimensions. The dashed line is $\Sigma$, comprised of links of the dual lattice. The plaquettes shown are those in $\Sigma^{*}$, which are dual to the links of $\Sigma$. The links marked by arrows are those in $\Omega^{*}$; they are dual to the plaquettes in a surface $\Omega$ that is bounded by $\Sigma$. (We may also interpret the dashed line as a slice through the closed surface $\Sigma$, in $3+1$ dimensions.)

where

$$
S_{\text {gauge }}=-\beta \sum_{P}\left(U_{P}+\text { c.c. }\right)
$$

and

$$
S_{\text {spin }}=-\sum_{m=1}^{N-1} \gamma_{m} \sum_{l}\left(\left[\left(\phi^{-1} U \phi\right)_{l}\right]^{m}+\text { c.c. }\right)
$$

Here $U_{P}=\prod_{l \in P} U_{l}$ associates with each elementary plaquette (labeled by $P$ ) the (ordered) product of the four $U_{l}$ associated with the (oriented) links of the plaquette, and $\left(\phi^{-1} U \phi\right)_{i j}=\phi_{i}^{-1} U_{i j} \phi_{j}$, for each pair $i j$ of nearest-neighbor sites. In eq. (4.3), we have introduced an independent spin coupling constant corresponding to each non-trivial irreducible representation of $\mathbb{Z}_{N}$.

Now we must consider how the operator $F(\Sigma)$ is to be defined in this lattice model *. Recall that inserting $F(\Sigma)$ into a Green function is supposed to be equivalent to introducing a classical cosmic string source on the world-sheet $\Sigma$. On the lattice (in $3+1$ dimensions), we consider $\Sigma$ to be a closed surface made up of plaquettes of the dual lattice. There is a set $\Sigma^{*}$ of plaquettes of the original lattice that are dual to the plaquettes of $\Sigma$. (See fig. 9.) The operator $F_{n}(\Sigma)$ may be expressed as

$$
F_{n}(\Sigma)=\prod_{P \in \Sigma^{*}} \exp \left(\beta\left(\mathrm{e}^{2 \pi i n / N} U_{P}-U_{P}+\text { c.c. }\right)\right)
$$

In other words, to evaluate the path integral for a Green function with an insertion of $F_{n}(\Sigma)$, we modify the plaquette action on the plaquettes that are dual to $\Sigma$, according to

$$
U_{P} \rightarrow \mathrm{e}^{2 \pi i n / N} U_{P}, \quad P \in \Sigma^{*}
$$

This transformation of $S_{\text {gauge }}$ is equivalent to introducing $n$ units of $\mathbb{Z}_{N}$ magnetic flux on each of the plaquettes in $\Sigma^{*}$.

* The analogous construction of the ' $t$ Hooft loop operator on the lattice was first discussed in refs. [33] and [34]. 
When the surface $\Sigma$ is large, the vacuum expectation value of $F_{n}$ behaves like

$$
\left\langle F_{n}(\Sigma)\right\rangle \sim \exp \left(-\kappa_{n}^{(\text {ren })} \operatorname{Area}(\Sigma)\right) .
$$

We may interpret $\kappa_{n}^{(\mathrm{ren})}$ as the renormalization of the tension of the string source, due to quantum fluctuations of the charged matter fields. Eq. (4.6) also has an alternative interpretation. We may think of $\Sigma$ as a surface lying in a time slice, rather than as the world-sheet of a string propagating through space-time. Then $F_{n}(\Sigma)$ is the operator

$$
F_{n}(\Sigma)=\exp \left(\frac{2 \pi i n}{N} Q_{\Sigma}\right)
$$

where $Q_{\Sigma}$ is the $\mathbb{Z}_{N}$ charge contained inside the surface $\Sigma$. Virtual pairs of charged particles near $\Sigma$ cause the charge $\mathrm{Q}_{\Sigma}$ to fluctuate. If the theory has a mass gap, then the charge fluctuations near two elements of $\Sigma$ become very weakly correlated when the elements are distantly separated. Thus, charge fluctuations cause the characteristic "area-law" falloff of $\left\langle F_{n}(\Sigma)\right\rangle$ in eq. (4.6).

Consider the case $\beta \gg 1$ and $\gamma_{m} \ll 1$ (for all $m$ ). In this case, the gauge variables are highly ordered, and so we expect that $\mathbb{Z}_{N}$ charge is not confined. Furthermore, the spins are disordered, so there is no Higgs condensate to screen $\mathbb{Z}_{N}$ charge either. Thus, free $\mathbb{Z}_{N}$ charges should exist. We can check whether this expectation is correct by using perturbative expansions to evaluate $\langle A(\Sigma, \mathrm{C})\rangle$.

Because the gauge coupling is weak, a frustrated plaquette (one with $U_{P} \neq 1$ ) is very costly. An insertion of $F_{n}(\Sigma)$ tends to frustrate the plaquettes in $\Sigma^{*}$. These frustrations can be avoided, though, if the gauge variables assume a suitable configuration. Choose a three-dimensional hypersurface $\Omega$ whose boundary is $\Sigma$. This hypersurface consists of a set of cubes of the dual lattice. Dual to these cubes is a set $\Omega^{*}$ of links of the original lattice. (See fig. 9.) By choosing

$$
\begin{array}{ll}
U_{l}=\mathrm{e}^{2 \pi i n / N}, & l \in \Omega^{*}, \\
U_{l}=1, & l \notin \Omega^{*},
\end{array}
$$

we can avoid frustrating any plaquettes. (This is the lattice analog of a "singular gauge transformation" that removes the string "singularity" on $\Sigma$.) With the links in the configuration eq. (4.8) the fundamental representation Wilson loop operator

$$
W(C)=\prod_{l \in C} U_{l}
$$

acquires the value $\exp (2 \pi i n k / N)$, where $k$ is the linking number of $C$ and $\Sigma$. (See fig. 10.) 


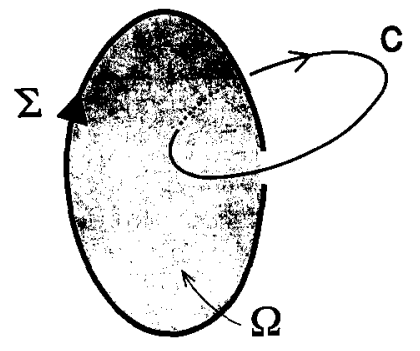

Fig. 10. A slice through a closed surface $\Sigma$ that links once with the loop $C$. $C$ intersects the hypersurface $\Omega$ once.

Of course, this choice of link variables changes the nearest neighbor interactions for each pair of spins that is joined by a link contained in $\Omega^{*}$. But the spins are strongly coupled and highly disordered, so that they are nearly indifferent to this change; it is much more costly to frustrate a plaquette than to frustrate a link. Therefore, the expectation value of the ABOP

$$
A_{n}^{(\nu)}(\Sigma, C)=\frac{F_{n}(\Sigma)[W(C)]^{\nu}}{\left\langle F_{n}(\Sigma)\right\rangle\left\langle[W(C)]^{\nu}\right\rangle}
$$

is dominated by small fluctuations about the "background" eq. (4.8), and we conclude that $\left\langle A_{1}^{(1)}(\Sigma, C)\right\rangle$ satisfies eq. (1.2). Thus, there is an infinite-range Aharonov-Bohm interaction, and free $\mathbb{Z}_{N}$ charges exist. (Further details of this analysis may be found in ref. [5].)

If the gauge coupling is strong $(\beta \ll 1)$, then there are no free $\mathbb{Z}_{N}$ charges. The Wilson loop introduces a $\mathbb{Z}_{N}$ charge as a classical source, but confinement causes a pair of $\mathbb{Z}_{N}$ charges to be produced, so that the charge is dynamically shielded. We expect that eq. (1.3) is satisfied, and this can be verified in the small- $\beta$ expansion [5].

It is also interesting to consider the case in which $\beta$ is large and some of the spin couplings $\gamma_{m}$ are also large. Then the matter field is ordered and the matter "condensate" screens the charge.

For example, suppose that

$$
\begin{aligned}
& \gamma_{m^{\prime}} \gg 1, \quad m^{\prime}=m, \\
& \gamma_{m^{\prime}} \ll 1, \quad m^{\prime} \neq m .
\end{aligned}
$$

In effect, then, the operator $\phi^{m}$ condenses, and $\mathbb{Z}_{N}$ is broken down to the kernel of the representation $(m)$; this is $\mathbb{Z}_{M}$, where $M$ is the greatest common factor of $N$ and $m$. We anticipate, therefore, that the operator $F_{n}(\Sigma)$ introduces a stable cosmic string world-sheet provided that $n m \equiv 0(\bmod N)$ (so that the flux carried 
by the string is in the unbroken group $\mathbb{Z}_{M}$ ). Otherwise, the string introduced by $F_{n}(\Sigma)$ is the boundary of a domain wall. This wall is unstable, and decays by nucleating a loop of string.

If we assume that the flux of the string created by $F_{n}(\Sigma)$ combines with the flux of the nucleated string to give a trivial total flux, then we anticipate that the ABOP behaves as

$$
\begin{array}{ll}
\lim \left\langle A_{n}^{(\nu)}(\Sigma, C)\right\rangle=\exp \left(\frac{2 \pi i n \nu}{N} k(\Sigma, C)\right), & m n \equiv 0(\bmod N), \\
\lim \left\langle A_{n}(\Sigma, C)\right\rangle=1, & m n \neq 0(\bmod N) .
\end{array}
$$

From this behavior, we could easily infer that the unbroken symmetry is $\mathbb{Z}_{M}$; a string has a non-trivial Aharonov-Bohm interaction with a charge if and only if the flux carried by the string is in $\mathbb{Z}_{M}$.

However, as we emphasized in subsect. 3.2, even if the string introduced by $F_{n}$ is the boundary of a domain wall, and the wall decays by nucleating a string loop, it need not be the case that the nucleated string combined with the classical string source has trivial flux. Thus, eq. (4.12) does not, in general, correctly describe the behavior of the ABOP in the limit eq. (4.11). To see what actually happens, let us analyze the consequences of eq. (4.11) using perturbative expansions.

For $\gamma_{m} \gg 1$, spins with nontrivial $\mathbb{Z}_{M}$ charge are highly ordered, and frustrating these spins is very costly. Now there is a competition between the reluctance of the system to frustrate a plaquette (when $\beta \gg 1$ ), and its reluctance to frustrate a $\mathbb{Z}_{M}$ spin. If the operator $F_{n}(\Sigma)$ is inserted, then, as we have seen, frustrated plaquettes can be avoided if the links of $\Omega^{*}$ are excited. But, if $\exp (2 \pi i n / N) \notin \mathbb{Z}_{M}$, we can excite the $U_{l}$ on these links only at the cost of frustrating the spins there. The number of links in $\Omega^{*}$ increases like the volume enclosed by $\Sigma$, and the number of plaquettes in $\Sigma^{*}$ increases only as the area of $\Sigma$. Thus, for $\Sigma$ sufficiently large, frustrated plaquettes are favored over frustrated links. This is just a realization on the lattice of the decay of a domain wall by nucleation of a loop string, where the frustrated spins comprise the wall, and the frustrated plaquettes comprise the nucleated string.

But as we discussed in subsect. 3.3, it is sometimes possible for a domain wall to decay in more than one way. To illustrate the possibilities, we will consider two different special cases.

4.1. $\mathbb{Z}_{4} \rightarrow \mathbb{Z}_{2}$

Consider, for example, a $\mathbb{Z}_{4}$ model. For $\beta \gg 1$ and $\gamma_{2} \gg 1$, the $\mathbb{Z}_{4}$ symmetry will break to $\mathbb{Z}_{2}$. One finds, indeed, that $\left\langle A_{2}^{(\nu)}(\Sigma, C)\right\rangle$ behaves as in eq. (4.12). 
Understanding the behavior of $A_{1}^{(\nu)}$ and $A_{3}^{(\nu)}$ involves a subtlety. The $n=3$ string is the anti-string of the $n=1$ string; therefore, they both have the same tension. Furthermore, $\exp (2 \pi i / 4)$ and $\exp (-2 \pi i / 4)$ belong to the same coset of $\mathbb{Z}_{2}$ in $\mathbb{Z}_{4}$, so the $n=1$ and $n=3$ strings are both boundaries of the same domain wall. When the operator $F_{1}(\Sigma)$ is inserted, the resulting domain wall can decay in two different ways. One way, the composite string that is created has trivial $\mathbb{Z}_{2}$ flux; the other way, it has non-trivial $\mathbb{Z}_{2}$ flux.

Correspondingly, when $F_{1}(\Sigma)$ is inserted, we may choose either $U_{l}=1$ or $U_{l}=-1$ on the links of $\Omega^{*}$; the weak spin coupling in the action (the $\gamma_{2}$ term) depends only on $U_{l}^{2}$, and so is not frustrated either way. But we also need to consider the dependence on the strong couplings $\gamma_{1}$ and $\gamma_{3}$. Expanding in these small parameters, one finds that the effective tension of the composite string is renormalized by spin fluctuations. The renormalization raises the tension of the composite string with non-trivial $\mathbb{Z}_{2}$ flux relative to that with trivial flux. Thus, the configuration such that the composite string has trivial $\mathbb{Z}_{2}$ flux really does dominate when $F_{1}(\Sigma)$ (or $F_{3}(\Sigma)$ ) is inserted. Therefore, $A_{1}^{(\nu)}$ and $A_{3}^{(\nu)}$ do behave as in eq. (4.12).

\section{2. $\mathbb{Z}_{6} \rightarrow \mathbb{Z}_{3}$}

Now consider a $\mathbb{Z}_{6}$ model. For $\beta \gg 1$ and $\gamma_{3} \gg 1$, the $\mathbb{Z}_{6}$ symmetry will break to $\mathbb{Z}_{3} . A_{2}^{(\nu)}$ and $A_{4}^{(\nu)}$ behave as in eq. (4.12); so do $A_{1}^{(\nu)}$ and $A_{5}^{(\nu)}$.

Now consider $A_{3}^{(\nu)}$. The $n=3$ string that is introduced by $F_{3}$ is the boundary of a domain wall. But this wall can terminate on an $n=1$ or $n=5$ string, as well as on an $n=3$ string. Furthermore, the $n=1$ (or $n=5$ ) string has lower tension than the $n=3$ string, so that nucleation of this string is favored.

In other words, the configurations that dominate, when $F_{3}(\Sigma)$ is inserted, have $U_{l}=\exp (2 \pi i / 3)$ (or $U_{l}=\exp (-2 \pi i / 3)$ ) for $l \in \Omega^{*}$. By exciting the links on $\Omega^{*}$, we reduce the degree of frustration of the plaquettes of $\Sigma$. And we do so at negligible cost, because the $m=3$ term in $S_{\text {spin }}$ cannot distinguish $U_{l}=$ $\exp ( \pm 2 \pi i / 3)$ from $U_{l}=1$.

Thus, the nucleated string, combined with the classical string source, has non-trivial flux. The combined flux can be either $n=2$ or $n=4$, and both occur with equal probability. And therefore, even though $F_{3}$ inserts a string that decays, $A_{3}$ shows non-trivial behavior; we have

$$
\lim \left\langle A_{3}^{(\nu)}(\Sigma, C)\right\rangle=\frac{1}{2}\left(\mathrm{e}^{2 \pi i \nu / 3}+\mathrm{e}^{-2 \pi i \nu / 3}\right)=\cos (2 \pi \nu / 3)
$$

(if $\Sigma$ and $C$ have linking number $k=1$ ).

Thus, for dynamical reasons, eq. (4.12) is not satisfied. Nevertheless, the behavior of $A_{n}^{(\nu)}$ has an unambiguous interpretation. For example, the $\nu$-dependence of $\left\langle A_{3}^{(\nu)}\right\rangle$ shows that the effective string introduced by $F_{3}$ has flux that takes 
values in the $\mathbb{Z}_{3}$ subgroup of $\mathbb{Z}_{6}$. The only possible interpretation is that the unbroken subgroup is $\mathbb{Z}_{3}$.

Indeed, the behavior of $\left\langle A_{n}^{(\nu)}\right\rangle$ always contains sufficient information to unambiguously identify the unbroken subgroup of an abelian group.

Other order parameters have been suggested that probe the realization of the center of the gauge group $[17,18,20]$. We will comment on the efficacy of these in subsect. 9.3.

\section{Non-abelian strings on the lattice}

We have seen in the previous section how the Aharonov-Bohm effect can be used to probe the phase structure of an abelian gauge theory. We now want to extend this procedure, so that it can be used in non-abelian theories. The basic strategy will be the same as before - we will introduce strings and charges as classical sources, and investigate the dynamical response of the theory to these sources. But the implementation of this strategy is more delicate in the non-abelian case. The main stumbling block is the problem of introducing non-abelian cosmic strings in a lattice gauge theory, which we address in this section.

We will also construct operators that create or annihilate dynamical string loops; correlation functions of these can be used to study the dynamical properties of strings.

\subsection{STRING CALIBRATION}

We consider a theory with (discrete) gauge group G. We are interested in how the local G-symmetry is realized. Specifically, we wish to identify the subgroup $H$ of $\mathrm{G}$ that admits free charges. ( $\mathrm{G}$ quantum numbers may be confined, or may be screened by a Higgs condensate.)

We investigate the realization of $G$ by assembling a laboratory that is equipped with cosmic string loops. As described in ref. [21] we can calibrate the string loops with a beam of particles that transform as some faithful (not necessarily irreducible) representation $(R)$ of $\mathrm{G}$. We choose an arbitrary basepoint $x_{0}$, and a basis for the representation $(R)$ at that point. We direct the beam from the basepoint to a beam splitter, allow the two beams to pass on either side of the string, and then recombine the beams and study the resultant interference pattern.

If the string is in a "group eigenstate" with flux (as defined in eq. (2.1)) $a \in \mathrm{G}$, and $|u\rangle$ is the wave function in internal-symmetry space of a particle at the basepoint, then, when the particle is transported around a closed path that begins and ends at $x_{0}$, the wave function is modified according to

$$
|u\rangle \rightarrow D^{(R)}(a)|u\rangle
$$


By observing the interference pattern, we can measure

$$
\left\langle u\left|D^{(R)}(a)\right| u\right\rangle
$$

By varying $|u\rangle$, we can then determine all matrix elements of $D^{(R)}(a)$, and hence $a$ itself. Given our choice of basepoint, and a choice of a basis for $D^{(R)}$, this procedure allows us to associate a well-defined group element with each string.

In the case where free G-charges may not exist, we must be careful that the separation between the two beams remains small compared to any confinement distance scale or Higgs screening length. At the same time, of course, the separation must be large compared to the thickness of the string core; we assume that the core is small compared to the characteristic distance scale of the dynamics that we wish to study.

Once we have calibrated the strings by measuring their Aharonov-Bohm interactions with nearby charges, we probe the dynamics of the theory by measuring the Aharonov-Bohm interactions of strings with distant charges. In this way, we hope to learn what quantum numbers are confined or screened, and to infer the "unbroken" subgroup $\mathrm{H}$.

(As we have noted, each string loop associated with an element of $G$ that is not in $\mathrm{H}$ will become the boundary of a domain wall. Thus it might seem that a good way to identify $\mathrm{H}$ is to observe which strings are attached to walls. Indeed, at sufficiently weak coupling, this is a sensible procedure, because the walls are very long lived. But at intermediate coupling this procedure may fail, because the walls decay rapidly by nucleating string loops. The time scale for the decay may be comparable to the time required to assemble and calibrate a string loop.)

Some problems with the calibration procedure should be pointed out. The first is that a string loop associated with a definite group element is not an eigenstate of the hamiltonian of the theory. An $a$-string and a $b$-string will mix with each other if $a$ and $b$ are in the same $\mathrm{H}$ conjugacy class [21], and the energy eigenstates will be linear combinations of group eigenstates that transform as definite representations of the unbroken group $\mathrm{H}$. (We will have more to say about this mixing in subsect. 7.2.) But the time scale for this mixing increases exponentially with the size of the string loop. For our purposes, it will usually be legitimate to ignore the mixing and regard the strings as group eigenstates.

But there is another more serious problem. While the quantum fluctuations that change the identity of a string loop are very rare (when the loop is large), there are other, much less rare, quantum fluctuations that can change the Aharonov-Bohm phase that is detected. Suppose, for example, that a virtual $b$-loop nucleates, lassoes the basepoint $x_{0}$ and then re-annihilates. Naturally, this process has no physical effect on an $a$-loop that is far from the basepoint. Yet it changes the Aharonov-Bohm phase acquired by a particle in representation $(\nu)$ that winds around the string, beginning and ending at $x_{0}$; we saw in subsect. 2.3 that the 


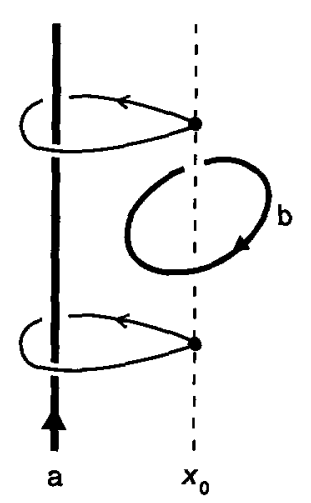

(a)

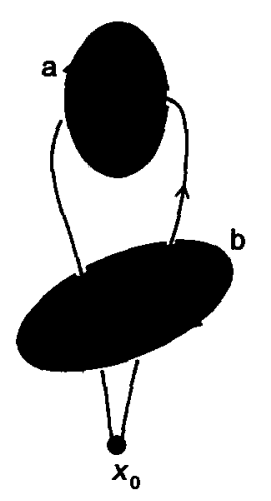

(b)

Fig. 11. (a) Space-time diagram (in $2+1$ dimensions) showing a virtual $b$ vortex-antivortex pair that nucleates, winds around the basepoint $x_{0}$, and re-annihilates. If the path based at $x_{0}$ at time $t_{1}$ is assigned the flux $a$, then the path at time $t_{2}$ is assigned flux $b a b^{-1}$. (b) The same process, but with $x_{0}$ now taken to be a fixed point in euclidean spacetime. The flux of the classical $a$-vortex is measured to $b a b^{-1}$, due to the effect of the virtual $b$ vortex pair. Shaded areas are surfaces bounded by the vortex world-lines.

phase becomes $D^{(\nu)}\left(b a b^{-1}\right)$ rather than $D^{(\nu)}(a)$. (This process is depicted, in $2+1$ dimensions, in fig. 11.) Such fluctuations are suppressed at sufficiently weak coupling, but they are present, at some level, for any finite coupling. They result in an ambiguity in the Aharonov-Bohm phase associated with a string, even if we fix the basepoint and a basis for the faithful representation $(\nu)$.

To avoid this ambiguity, we are forced to take a trace of the representation; the character $\chi^{(\nu)}(a)$ is unaffected by these fluctuations. Thus, the existence of free G-charges (and of an unscreened Aharonov-Bohm interaction) can be probed by the Wilson loop operator eq. (2.5). (And since $W^{(\nu)}(C)$ is gauge invariant, there is no need for the loop $C$ to contain the basepoint $x_{0}$.) Let $F_{a}\left(\Sigma, x_{0}\right)$ denote the operator that inserts a string world-sheet on $\Sigma$; the string is associated with $a \in \mathrm{G}$, relative to the basepoint $x_{0}$. (We consider below how this operator is constructed.) Then we may define the ABOP

$$
A_{a}^{(\nu)}\left(\Sigma, x_{0} ; C\right)=\frac{F_{a}\left(\Sigma, x_{0}\right) W^{(\nu)}(C)}{\left\langle F_{a}\left(\Sigma, x_{0}\right)\right\rangle\left\langle W^{(\nu)}(C)\right\rangle} .
$$

In a phase with free G-charges, we expect that [14]

$$
\lim \left\langle A_{a}^{(\nu)}\left(\Sigma, x_{0} ; C\right)\right\rangle=\frac{1}{n_{\nu}} \chi^{(\nu)}\left(a^{k(\Sigma, C)}\right),
$$

where $k$ denotes the linking number. (Even this statement requires a qualification, 
for the loop $C$ must not be permitted to come close to retracing itself on successive passages around $\Sigma$.)

We will consider in section 9 how $A_{a}^{(\nu)}\left(\Sigma, x_{0} ; C\right)$ is expected to behave for other realizations of the local G-symmetry.

\subsection{INSERTING STRING WORLD-SHEETS}

Now we will consider in more detail the problem of constructing an operator that inserts non-abelian strings on specified world-sheets. The construction will be guided by the discussion in sect. 2 of the general formalism, and by the discussion above of the calibration procedure.

Given a set of disjoint closed two-dimensional surfaces $\Sigma_{1}, \Sigma_{2} \ldots$, this operator is to introduce string world-sheets on these surfaces, where the strings are associated with the group elements $a_{1}, a_{2}, \ldots$. As we have emphasized, the strings must be referred to a common basepoint $x_{0}$, and each associated group element depends on the choice of a standard path that begins and ends at $x_{0}$ and winds around the string. The group elements $a_{1}, a_{2}, \ldots$ are defined up to one overall conjugation, which corresponds to a gauge transformation at the basepoint $x_{0}$.

Furthermore, the definition of our operator must be insensitive to the possible confinement or screening of $\mathrm{G}$ quantum numbers; it must specify the short-distance structure of the string, and leave it up to the dynamics of the theory whether the string can be detected at long range. It must realize the calibration procedure described above, in which the short-range Aharonov-Bohm interactions of the string are determined.

We wish to define an operator $F_{a_{1}, a_{2}, \ldots, a_{n}}\left(\Sigma_{1}, \Sigma_{2}, \ldots, \Sigma_{n}, x_{0}\right)$ so that an insertion of $F_{a_{1}, a_{2}, \ldots, a_{n}}\left(\Sigma_{1}, \Sigma_{2}, \ldots, \Sigma_{n}, x_{0}\right)$ in a Green function introduces strings on world sheets $\Sigma_{1}, \Sigma_{2}, \ldots, \Sigma_{n}$. We will first describe the operator in heuristic terms, and then give a more precise description in the context of lattice gauge theory. Loosely speaking, an insertion of $F_{a_{1}, a_{2}, \ldots, a_{n}}\left(\Sigma_{1}, \Sigma_{2}, \ldots, \Sigma_{n}, x_{0}\right)$ in a Green function imposes a restriction on the gauge field configurations that are included in the path integral. Make a choice of a standard path that begins at $\mathrm{x}_{0}$, winds around the surface $\Sigma_{1}$, and returns to $x_{0}$. Now consider a path $P_{1}$ homotopic to the standard path that runs from $x_{0}$ to a point on $\Sigma_{1}$, traverses an infinitesimal closed loop that encloses $\Sigma_{1}$, and then retraces itself, returning to $x_{0}$. When $F_{a_{1}, a_{2}, \ldots, a_{n}}\left(\Sigma_{1}\right.$, $\left.\Sigma_{2}, \ldots, \Sigma_{n}, x_{0}\right)$ is inserted, the gauge field configuration is restricted to satisfy

$$
P \exp \left(i \oint_{P_{1}, x_{0}} A \cdot \mathrm{d} x\right)=a_{1},
$$

for any such $P_{1}$. Similar restrictions apply for paths $P_{2}, P_{3}, \ldots, P_{n}$ that wind around $\Sigma_{2}, \Sigma_{3}, \ldots, \Sigma_{n}$, as in fig. 12. These restrictions on the path integral define the operator $F_{a_{1}, a_{2}, \ldots, a_{n}}\left(\Sigma_{1}, \Sigma_{2}, \ldots, \Sigma_{n}, x_{0}\right)$. 


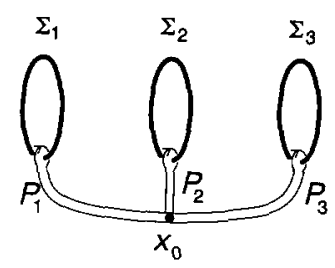

Fig. 12. Paths $P_{1}, P_{2}, P_{3}$ used in the construction of the operator $F_{a_{1}, a_{2}, a_{3}}\left(\Sigma_{1}, \Sigma_{2}, \Sigma_{3} ; x_{0}\right)$.

The operator so constructed is not gauge invariant. Of course, if it is inserted in a Green function with gauge invariant operators, its gauge-invariant part will be projected out. Alternatively we may obtain an explicitly gauge-invariant operator by averaging over gauge transformations at the basepoint $x_{0}$, obtaining

$$
\frac{1}{n_{\mathrm{G}}} \sum_{g \in \mathrm{G}} F_{g a_{1} g^{-1}, g a_{2} g^{-1}, \ldots, g a_{n} g^{-1}}\left(\Sigma_{1}, \Sigma_{2}, \ldots, \Sigma_{n}, x_{0}\right),
$$

where $n_{\mathrm{G}}$ is the order of the group.

We may now consider the operator

$$
\begin{aligned}
& A_{a_{1}, a_{2}, \ldots, a_{n}}^{(v)}\left(\Sigma_{1}, \Sigma_{2}, \ldots, \Sigma_{n}, x_{0} ; C\right) \\
& \quad=\frac{F_{a_{1}, a_{2}, \ldots, a_{n}}\left(\Sigma_{1}, \Sigma_{2}, \ldots, \Sigma_{n}, x_{0}\right) W^{(\nu)}(C)}{\left\langle F_{a_{1}, a_{2}, \ldots, a_{n}}\left(\Sigma_{1}, \Sigma_{2}, \ldots, \Sigma_{n}, x_{0}\right)\right\rangle\left\langle W^{(\nu)}(C)\right\rangle} .
\end{aligned}
$$

In a phase with free G-charge, if the loop $C$ is homotopic to $P_{i} P_{j} P_{k} \ldots$, we have

$$
\lim \left\langle A_{a_{1}, a_{2}, \ldots, a_{n}}^{(\nu)}\left(\Sigma_{1}, \Sigma_{2}, \ldots, \Sigma_{n}, x_{0} ; C\right)\right\rangle=\frac{1}{n_{\nu}} \chi^{(\nu)}\left(a_{i} a_{j} a_{k} \ldots\right)
$$

Thus, when a charge passes around several string loops in succession, the Aharonov-Bohm phases acquired in each successive passage are combined coherently. The coherence is maintained because we have defined the various loops in reference to the same basepoint $x_{0}$. If different basepoints had been chosen instead, then the group elements associated with the various string loops would have been averaged over conjugacy classes independently. Since

$$
\frac{1}{n_{\mathrm{G}}} \sum_{g \in \mathrm{G}} D^{(\nu)}\left(\mathrm{gag}^{-1}\right)=\frac{1}{n_{\nu}} \chi^{(\nu)}(a) \mathbb{I}
$$


(which follows from Schur's lemma), we find, for example,

$$
\lim \frac{\left\langle F_{a_{1}}\left(\Sigma_{1}, x_{0}\right) F_{a_{2}}\left(\Sigma_{2}, y_{0}\right) W^{(\nu)}(C)\right\rangle}{\left\langle F_{a_{1}}\left(\Sigma_{1}, x_{0}\right) F_{a_{2}}\left(\Sigma_{2}, y_{0}\right)\right\rangle\left\langle W^{(\nu)}(C)\right\rangle}=\frac{1}{n_{\nu}} \chi^{(\nu)}\left(a_{1}\right) \frac{1}{n_{\nu}} \chi^{(\nu)}\left(a_{2}\right),
$$

if the loop $C$ winds around world-sheets $\Sigma_{1}$ and $\Sigma_{2}$ in succession.

If $F_{a_{1}}\left(\Sigma_{1}, x_{0}\right)$ and $F_{a_{2}}\left(\Sigma_{2}, y_{0}\right)$ have distinct basepoints $\left(x_{0} \neq y_{0}\right)$, then each by itself introduces a gauge-singlet object. Inserting both operators combines two string loops as (trivial) charge eigenstates, rather than as group eigenstates. This is the reason for the lack of coherence in the Aharonov-Bohm interaction characterized by eq. (5.10). To combine two strings that are not both gauge singlets, we must include in our operator some non-local construct that bridges the gap between the string loops, just as a string of electric flux must be included in a gauge-invariant operator that creates a widely separated quark-antiquark pair. In the case of two (or more) string loops, this non-local connection between the loops is provided by referring the loops to a common basepoint.

It is important, actually, that the Aharonov-Bohm interactions of an additional loop combine incoherently with the Aharonov-Bohm interactions of existing loops, when the group element associated with the additional loop is averaged over a conjugacy class. It is this incoherence property that ensures that the effects of virtual string loops do not spoil the "factorization up to a phase" represented by eq. (5.8).

We will now describe the construction of $F_{a}\left(\Sigma, x_{0}\right)$ in more detail, by specifying how the construction is carried out in a lattice theory with discrete gauge group $\mathrm{G}$. As discussed in sect. $4, \Sigma$ is to be regarded as a closed surface consisting of plaquettes of the dual lattice; these plaquettes are dual to a set $\Sigma^{*}$ of plaquettes of the original lattice. For each plaquette $P \in \Sigma^{*}$, we choose a path $l_{P}$ on the lattice that connects the basepoint $x_{0}$ to one of the corners of $P$. These paths are chosen so that each closed loop $l_{P} P l_{P}^{-1}$ is "homotopic" to the standard loop that links $\Sigma$. The various paths may be chosen arbitrarily, except that the union of all the paths should not contain any closed loops.

The effect of the operator $F_{a}\left(\Sigma, x_{0}\right)$ is to modify the plaquette action on each plaquette in $\Sigma^{*}$. Suppose, for example, that the plaquette action is

$$
S_{\text {gauge }, P}^{(R)}=-\beta \chi^{(R)}\left(U_{P}\right)+\text { c.c. }
$$

(where $R$ is a representation of $\mathrm{G}$ that must be specified to define the theory). Then an insertion of $F_{a}\left(\Sigma, x_{0}\right)$ modifies the action according to

$$
S_{\text {gauge }, P}^{(R)} \rightarrow-\beta \chi^{(R)}\left(V_{l_{P}} a V_{l_{P}}^{-1} U_{P}\right)+\text { c.c., } \quad P \in \Sigma^{*},
$$


where

$$
V_{l_{P}}=\prod_{l \in l_{P}} U_{l}
$$

Alternatively, we may write

$$
F_{a}\left(\Sigma, x_{0}\right)=\prod_{P \in \Sigma^{*}} \exp \left(\beta \chi^{(R)}\left(V_{l_{P}} a V_{l_{P}}^{-1} U_{P}\right)-\beta \chi^{(R)}\left(U_{P}\right)+\text { c.c. }\right) .
$$

The operator $F_{a_{1}, a_{2}, \ldots, a_{n}}\left(\Sigma_{1}, \Sigma_{2}, \ldots, \Sigma_{n}, x_{0}\right)$ that inserts many string loops is constructed by a straightforward generalization of this procedure.

As constructed, $F_{a}\left(\Sigma, x_{0}\right)$ is not gauge invariant. When inserted in a Green function with gauge-invariant operators, though, it has the same effect as the explicitly gauge-invariant operator in which $a$ is averaged over a conjugacy class, as in eq. (5.6).

It is also instructive to consider the correlator of $F_{a}\left(\Sigma, x_{0}\right)$ with the operator

$$
U^{(\nu)}\left(C, x_{0}\right)=D^{(\nu)}\left(\prod_{l \in C} U_{l}\right)
$$

where the product is taken over a closed set of links that begins and ends at $x_{0}$. The trace of $U^{(\nu)}\left(C, x_{0}\right)$ is $\left(n_{\nu}\right.$ times) the Wilson loop operator $W^{(\nu)}(C)$. But $U^{(\nu)}\left(C, x_{0}\right)$ itself, like $F_{a}\left(\Sigma, x_{0}\right)$, is not invariant under a gauge transformation that acts non-trivially at the basepoint $x_{0}$.

In a phase with free G-charges, and in the leading order of weak coupling perturbation theory, one finds that *

$$
\lim \frac{\left\langle F_{a}\left(\Sigma, x_{0}\right) U^{(\nu)}\left(C, x_{0}\right)\right\rangle}{\left\langle F_{a}\left(\Sigma, x_{0}\right)\right\rangle\left\langle\operatorname{tr} U^{(\nu)}\left(C, x_{0}\right)\right\rangle}=\frac{1}{n_{\nu}} D^{(\nu)}\left(a^{k(\Sigma, C)}\right) .
$$

This equation merely states that, once a loop of string has been calibrated, the same Aharonov-Bohm phase can be recovered again if another interference experiment is subsequently performed. But we have already emphasized that quantum fluctuations (such as the virtual string depicted in fig. 11) can spoil this result. Indeed, when higher orders in the weak coupling expansion are included, it is seen that the correlator of $F_{a}\left(\Sigma, x_{0}\right)$ and $U^{(v)}\left(C, x_{0}\right)$ fails to "factorize" as in eq. (5.16), even when $\Sigma$ and $C$ are far apart. As stated before, we must consider the correlator of $F_{a}\left(\Sigma, x_{0}\right)$ with the gauge-invariant operator $W^{(\nu)}(C)$, in order to extract an Aharonov-Bohm "phase" that depends only on the topological linking of $\Sigma$ and $C$ (in the limit of infinite separation).

- This calculation is described in more detail in subsect. 6.2 and in appendix A. 


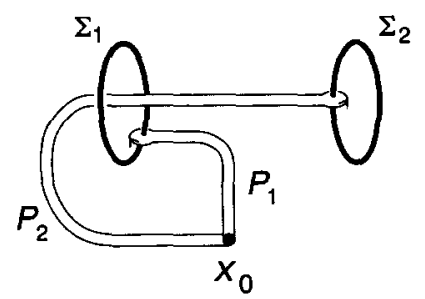

Fig. 13. A different choice for the path $P_{2}$ from the basepoint $x_{0}$ to the world-sheet $\Sigma_{2}$.

To conclude this section, we must ask how the operator $F_{a}\left(\Sigma, x_{0}\right)$ depends on the basepoint $x_{0}$ and on the choice of the paths $\left\{l_{P}\right\}$. (We consider correlation functions of $F_{a}$ with gauge-invariant operators, so we regard $F_{a}$ as gauge-invariant, with $a$ averaged over a conjugacy class.) We note that, since the union $U_{P} l_{P}$ of all the paths contains no closed loops, we can choose a gauge with $U_{l}=1$ for all $l \in l_{P}$. In this gauge, the group element $a$ is inserted directly on the plaquettes of $\Sigma^{*}$. Thus, it is clear that the correlator of $F_{a}\left(\Sigma, x_{0}\right)$ with any local gauge-invariant operator is independent of the choice of basepoint and paths.

The correlator of $F_{a_{1}, a_{2}, \ldots, a_{n}}\left(\Sigma_{1}, \Sigma_{2}, \ldots, \Sigma_{n}, x_{0}\right)$ with nonlocal gauge-invariant operators (like Wilson loop operators) depends on the choice of path only to the extent that we have already noted. That is, it depends on the choice of the "standard paths" that enter the calibration of the string loops. We may change the paths from the basepoint $x_{0}$ to the plaquettes of $\Sigma_{2}^{*}$, by winding these paths around $\Sigma_{1}$, as in fig. 13. In effect, this change alters the group element assigned to the string world sheet on $\Sigma_{2} ; a_{2}$ becomes replaced by $a_{1} a_{2} a_{1}^{-1}$. As we noted in sect. 2, this ambiguity in $F_{a_{1}, a_{2}, \ldots, a_{n}}\left(\Sigma_{1}, \Sigma_{2}, \ldots, \Sigma_{n}, x_{0}\right)$ is the origin of the holonomy interaction between string loops (or vortices) [24,25].

\subsection{INSERTING STRING LOOPS}

An insertion of the operator $F_{a}\left(\Sigma, x_{0}\right)$ introduces a string on the closed world-sheet $\Sigma$. The string may be regarded as an infinitely heavy classical source. Thus $F_{a}\left(\Sigma, x_{0}\right)$ is closely analogous to the Wilson loop operator $W^{(\nu)}(C)$; an insertion of $W^{(\nu)}(C)$ introduces an infinitely heavy classical source (in representation $(\nu))$ on the closed world line $C$.

But we will also find use at times for an operator that creates (or annihilates) a dynamical cosmic string. Such an operator can be obtained by a simple modification of the construction described above - namely, the surface $\Sigma$ is chosen to be, rather than a closed surface, a surface with non-trivial boundary $C$. (See fig. 14.) Carrying out the same procedure as before for such a surface, we arrive at an operator $B_{a}\left(C, \Sigma, x_{0}\right)$ that creates a string (or annihilates an anti-string) on the 


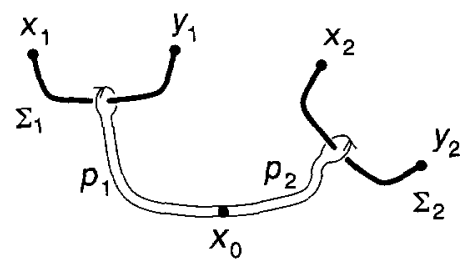

Fig. 14. The paths $P_{1}$ and $P_{2}$, based at $x_{0}$, and the open curves $\Sigma_{1}$ and $\Sigma_{2}$ used in the definition of the 't Hooft operator $B_{a, b}\left(x_{1}, y_{1}, \Sigma_{1}, x_{2}, y_{2}, \Sigma_{2}, x_{0}\right)$, in $2+1$ dimensions.

loop C. More generally, an operator

$$
B_{a_{1}, a_{2}, \ldots, a_{n}}\left(C_{1}, \Sigma_{1}, C_{2}, \Sigma_{2}, \ldots, C_{n}, \Sigma_{n}, x_{0}\right)
$$

creates strings on the loops $C_{1}, C_{2}, \ldots, C_{n}$, with all strings referred to a common basepoint $x_{0}$.

This construction generalizes a construction devised by 't Hooft [16], and we will refer to $B$ as the "'t Hooft loop" operator. However, 't Hooft considered the situation in which the strings have no Aharonov-Bohm interactions with other fields. In that case, the surface $\Sigma$ is an invisible gauge artifact, and $B(C)$ depends on $C$ alone. We are interested in strings that have Aharonov-Bohm interactions, and in that case $\Sigma$ is not invisible [5].

To better understand why the 't Hooft operator $B_{a}\left(C, \Sigma, x_{0}\right)$ must depend on the surface $\Sigma$ as well as on the loop $C$, it is helpful to think about a theory defined in $2+1$ space-time dimensions. In that case, the operator $B_{a}\left(x, y, \Sigma, x_{0}\right)$ creates a vortex at $x$ and an anti-vortex at $y ; \Sigma$ is a path connecting $x$ and $y$. But no gauge-invariant local operator exists that creates an isolated vortex at $x$. Because a vortex can be detected at infinite range via the Aharonov-Bohm effect, there is a vortex superselection rule. Hence, the operator that creates a vortex cannot be local; it has a semi-infinite string that can be seen by the fields of the theory. (Similarly, there is an electric charge superselection rule in quantum electrodynamics. No gauge-invariant local operator can create an isolated electron; the operator that creates an electron must also create a string of electric flux that ends on the electron.)

Correlation functions such as $\left\langle B_{a_{1}, a_{2}}\left(C_{1}, \Sigma_{1}, C_{2}, \Sigma_{2}, x_{0}\right)\right\rangle$ can be used to determine the tension of a dynamical string, or the amplitude for mixing between group eigenstates, as we will describe in section 7.

(As an aside, we remark that an 't Hooft loop, like a Wilson loop, admits an alternative interpretation. If a Wilson loop operator acts on a time-like slice, it is natural to interpret it as an insertion of a classical charged source, as noted above. But if the Wilson loop acts on a space-like slice, we may interpret it as an operator that creates a closed electric flux tube. We have noted that we may think of an 't Hooft operator acting on a space-like slice as an object that creates a cosmic string. 
Alternatively, we may interpret an 't Hooft loop acting on a time-like slice as an insertion of a classical magnetic monopole source. In the situation originally considered by 't Hooft, the monopole satisfied the Dirac quantization condition, and so its "Dirac string" was invisible. We are considering a situation in which the Dirac string is visible; the surface $\Sigma$ bounded by $C$ is the world sheet of this Dirac string.)

\section{Classical strings in the pure gauge theory}

We will now analyze the behavior of the operator $A_{a}^{(\nu)}\left(\Sigma, x_{0} ; C\right)$, using perturbative expansions. Here we will consider the case of a pure gauge theory with (discrete) gauge group G. In sect. 9, we will consider the effects of introducing matter.

\subsection{STRONG COUPLING}

Although our main interest is in the physics at weak gauge coupling, we will make a few comments about the strong-coupling behavior of the pure gauge theory.

The plaquette action of the theory is taken to be

$$
S_{\text {gauge }, P}^{(R)}=-\beta \chi^{(R)}\left(U_{P}\right)+\text { c.c., }
$$

where $R$ is a representation of $\mathrm{G}$. For $\beta \ll 1$, this theory confines sources that transform as certain irreducible representations of $\mathrm{G}$. The criterion for a source to be confined is easiest to state in the case where $R$ is irreducible. In that case, a source in the irreducible representation $(\nu)$ is not confined if and only if there are non-negative integers $k_{1}$ and $k_{2}$ such that

$$
\begin{aligned}
& (R)^{k_{1}} \otimes\left(R^{*}\right)^{k_{2}} \supset(\nu), \\
& (R)^{k_{1}} \otimes\left(R^{*}\right)^{k_{2}} \supset(1),
\end{aligned}
$$

where $R^{*}$ denotes the complex conjugate of $R$, and (1) denotes the trivial representation. The point is that if eq. (6.2) is satisfied, then it is possible for a source in representation $(\nu)$ to be "screened by gluons." In other words, the electric flux tube that terminates on a $(\nu)$ source can break due to glue fluctuations. (The criterion (6.2) generalizes the familiar notion that an adjoint representation source is unconfined in a strongly coupled $\mathrm{SU}(N)$ gauge theory.) If $(\nu)$ is 
confined, then the expectation value of the corresponding Wilson loop operator decays for a large loop $C$ like

$$
\left\langle W^{(\nu)}(C)\right\rangle \sim \exp \left(-\kappa^{(\nu)} \operatorname{Area}(C)\right),
$$

where $\operatorname{Area}(C)$ is the minimal area of a surface bounded by $C$, and $\kappa^{(\nu)}$ is the tension of a flux tube that carries electric flux in the representation ( $\nu$ ). (Of course, virtual glue may partially screen the source; $\kappa^{(\nu)}$ is the tension of the lightest flux tube that can terminate on a $(\nu)$ source.)

Since the gauge variables can see a classical string with "magnetic flux" $a \in \mathrm{G}$, the quantum fluctuations of the gauge variables renormalize the tension of the string by an amount $\kappa_{a}^{(\text {ren })}$. Thus, the expectation value of the operator $F$ decays for a large surface $\Sigma$ like

$$
\left\langle F_{a}\left(\Sigma, x_{0}\right)\right\rangle \sim \exp \left(-\kappa^{(\mathrm{ren})} A(\Sigma)\right),
$$

where $A(\Sigma)$ is the area of $\Sigma$. The calculation of the leading contribution to $\kappa_{a}^{(\mathrm{ren})}$, for $\beta \ll 1$, is described in appendix $\mathrm{A}$.

Now consider the operator $A_{a}^{(\nu)}\left(\Sigma, x_{0} ; C\right)$. Since there are no infinite-range Aharonov-Bohm interactions in the confining phase of the theory, we might expect that

$$
\lim \left\langle A_{a}^{(\nu)}\left(\Sigma, x_{0} ; C\right)\right\rangle=1 .
$$

This is indeed found for a representation $(\nu)$ that is not confined (such that the electric flux tube can break). Different behavior is found, however, for a representation $(\nu)$ that is confined. The operator $A_{a}^{(\nu)}\left(\Sigma, x_{0} ; C\right)$ can have a non-trivial expectation value because the electric flux tube stretched across $C$ crosses the surface $\Sigma$ at certain points. In the leading order of strong-coupling perturbation theory (and assuming that no "partial screening" of the source occurs), each crossing contributes to $A$ the factor

$$
\frac{1}{n_{\nu}} \chi^{(\nu)}(a)
$$

(or its complex conjugate, depending on the relative orientation of the flux tube and $\Sigma$ at the point of crossing). Thus, even when $\Sigma$ and $C$ are far apart, $\left\langle A_{a}^{(\nu)}\left(\Sigma, x_{0} ; C\right)\right\rangle$ is not a purely topological quantity that depends only on the linking number of $\Sigma$ and $C$. Of course, in higher orders in the strong coupling expansion, the behavior of $A_{a}^{(\nu)}$ becomes still more complicated.

Similarly, if a loop $C$ links with two different surfaces $\Sigma_{1}$ and $\Sigma_{2}$, the operator $A_{a_{1}, a_{2}}^{(\nu)}\left(\Sigma_{1}, \Sigma_{2}, x_{0} ; C\right)$ acquires a factor $\left(1 / n_{\nu}\right) \chi^{(\nu)}\left(a_{1}\right)$ each time the electric flux tube crosses $\Sigma_{1}$ and a factor $\left(1 / n_{\nu}\right) \chi^{(\nu)}\left(a_{2}\right)$ each time it crosses $\Sigma_{2}$ (in the leading 
order of the strong-coupling expansion). Because of confinement, the string world-sheets combine as trivial charge eigenstates rather than group eigenstates, even though both are defined with respect to a common basepoint.

\subsection{WEAK COUPLING}

For $\beta \gg 1$ there is no confinement, and the Wilson loop operator exhibits perimeter law decay for any representation $(\nu)$.

The operator $F_{a}\left(\Sigma, x_{0}\right)$ decays as in eq. (6.4). The calculation of the leading behavior of $\kappa_{a}^{(\text {ren })}$, for $\beta \gg 1$, is described in appendix $\mathrm{A}$.

When the operator $F_{a}\left(\Sigma, x_{0}\right)$ is inserted, there is a configuration of the gauge variables such that no plaquettes are excited. This configuration can be constructed by choosing a set $\Omega$ of cubes of the dual lattice such that the boundary of $\Omega$ is $\Sigma$. Each cube in $\Omega$ is dual to a link of the original lattice. The configuration with no excited plaquettes is

$$
\begin{aligned}
& U_{l}=a \quad l \in \Omega^{*}, \\
& U_{l}=e, \quad l \notin \Omega^{*} .
\end{aligned}
$$

This configuration is unique up to a gauge transformation. (The gauge transformations are deformations of $\Omega$.)

Weak-coupling perturbation theory is carried out by expanding in the number of excited plaquettes, and in the degree of excitation. In the limit $\beta \rightarrow \infty$, the configurations with the minimal number of excited plaquettes dominate. By calculating the Wilson loop operator for the configuration with no excited plaquettes, we verify eq. (5.4) in the weak-coupling limit. Thus we find, as expected, that G-charges are neither confined nor screened. Similarly, we may verify eq. (5.8) and eq. (5.16) in this limit.

When higher-order corrections in weak-coupling perturbation theory are computed, we find as anticipated that eq. (5.4) and eq. (5.8) continue to hold. But eq. (5.16) does not survive. These corrections are further discussed in appendix A.

We wish to make one other remark here about the weak-coupling expansion, which might help to avoid confusion. To calculate the weak-coupling behavior of correlation functions that involve the operator $F_{a_{1}, a_{2}, \ldots, a_{n}}\left(\Sigma_{1}, \Sigma_{2}, \ldots, \Sigma_{n}, x_{0}\right)$, we first construct the configuration that has no excited plaquettes when $F_{a_{1}, a_{2}, \ldots, a_{n}}\left(\Sigma_{1}, \Sigma_{2}, \ldots, \Sigma_{n}, x_{0}\right)$ is inserted. This construction is a straightforward generalization of eq. (6.7). However, there are topologically inequivalent ways of choosing non-intersecting surfaces $\Omega_{1}, \ldots, \Omega_{n}$, that are bounded by $\Sigma_{1}, \ldots, \Sigma_{n}$ (as in fig. 15). Thus, one might get the impression that there can be two (or more) gauge-inequivalent configurations that both have no excited plaquettes. But this is not the case. To see why not, it is important to keep track of the basepoint, and of the paths from the basepoint to the loops. If $\Omega_{1}$ is distorted past $\Omega_{2}$, as in fig. 15 , 


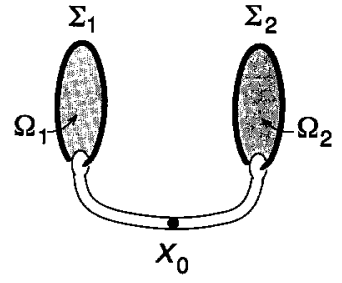

(a)

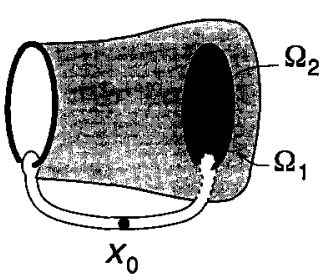

(b)

Fig. 15. Two topologically inequivalent ways of choosing the hypersurface $\Omega_{1}$ that is bounded by $\Sigma_{1}$.

then $\Omega_{1}$ crosses the paths from the basepoint to $\Sigma_{2}$. To avoid exciting any plaquettes, then, the links contained in $\Omega_{2}^{*}$ must now take the value $a_{1} a_{2} a_{1}^{-1}$. (This, again, is a reflection of the holonomy interaction between string loops.) Therefore, a Wilson loop that crosses $\Omega_{1}$ first and $\Omega_{2}$ second, in fig. 15a, behaves exactly the same way as a Wilson loop that crosses $\Omega_{2}$ first and $\Omega_{1}$ second, in fig. $15 \mathrm{~b}$. There is a unique gauge equivalence-class of configurations with no excited plaquettes, just as there should be.

\section{Dynamical strings and vortices}

We will now consider how the 't Hooft operator can be used to investigate the properties of dynamical strings (in $3+1$ dimensions) and vortices (in $2+1$ dimensions).

\subsection{STRING TENSION AND VORTEX MASS}

The operator $B_{a, a^{-1}}\left(C_{1}, \Sigma_{1}, C_{2}, \Sigma_{2}, x_{0}\right)$ can be used to compute the tension of a cosmic string that carries magnetic flux $a$ (with a caveat described below). This operator creates an $a$ string on $C_{1}$ and annihilates it on $C_{2}$. Thus, when the loops are large and far apart, we have

$$
\begin{aligned}
& \left\langle B_{a, a^{-1}}\left(C_{1}, \Sigma_{1}, C_{2}, \Sigma_{2}, x_{0}\right)\right\rangle \\
& \quad \sim \exp \left(-\kappa_{a}^{\text {(ren })}\left(A\left(\Sigma_{1}\right)+A\left(\Sigma_{2}\right)\right)\right) \exp \left(-\kappa_{a}^{\text {(dyn) }} A\left(C_{1}, C_{2}\right)\right) .
\end{aligned}
$$

Here $\kappa_{a}^{(\mathrm{ren})}$ is the renormalization of the tension of a "classical" string source, and $\kappa_{a}^{(\text {dyn })}$ is the tension of a dynamical string; $A\left(C_{1}, C_{2}\right)$ is the area of the minimal surface with boundary $C_{1} \cup C_{2}$. (See fig. 16.) If the loops $C_{1}$ and $C_{2}$ are chosen to be far apart compared to the correlation length of the theory, but close together compared to the size of the loops, then the dependence of $\left\langle B_{a, a^{-1}}\left(C_{1}, \Sigma_{1}, C_{2}\right.\right.$, $\left.\left.\Sigma_{2}, x_{0}\right)\right\rangle$ on the separation between the loops determines the tension $\kappa_{a}^{\text {(dyn) }}$. 


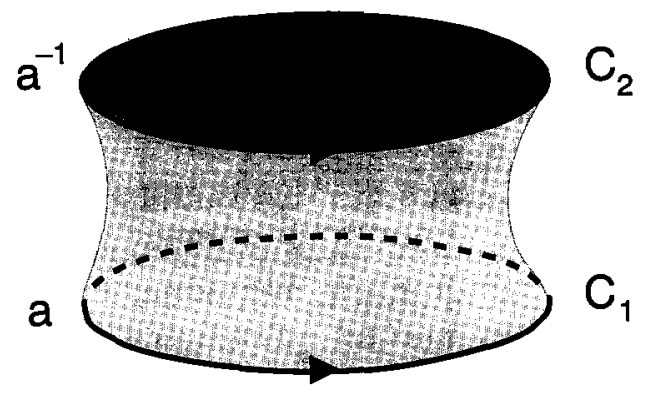

Fig. 16. Surface of minimal area bounded by the loop $C_{1}$ and $C_{2}$ (when the loops are close together).

Actually, the same information can be extracted from the behavior of the simpler operator $B_{a}\left(C, \Sigma, x_{0}\right)$. For a large loop $C$, we have

$$
\left\langle B_{a}\left(C, \Sigma, x_{0}\right)\right\rangle \sim \exp \left(-\kappa_{a}^{(\mathrm{ren})} A(\Sigma)\right) \exp \left(-\kappa_{a}^{(\mathrm{dyn})} A(C)\right),
$$

where $A(C)$ is the area of the minimal surface bounded by $C$. (See fig. 17.) Since $A(\Sigma)$ and $A(C)$ can be varied independently, $\kappa_{a}^{\text {(dyn) }}$ can be determined. (Or, $F_{a}\left(\Sigma, x_{0}\right)$ can be used to measure $\kappa_{a}^{(\mathrm{ren})}$.) The calculation of $\kappa_{a}^{\text {(dyn) }}$ in weak-coupling perturbation theory is described in appendix A. (In the strong-coupling limit, we have $\kappa^{(\mathrm{dyn})}=0$ - there are no stable magnetic flux tubes.)

Obviously, the same procedure can be used to calculate the mass of a vortex, in $2+1$ dimensions.

The existence of a stable string (or vortex) can itself be used to probe the phase structure of the theory. In a confining phase, stable magnetic flux tubes do not exist; they "melt" due to magnetic disorder. If the G gauge symmetry is spontaneously broken to a subgroup $\mathrm{H}$, stable $a$ strings exist only if $a \in \mathrm{H}$. Otherwise, an $a$ string is the boundary of a domain wall, which decays as described in subsect. 3.2 .

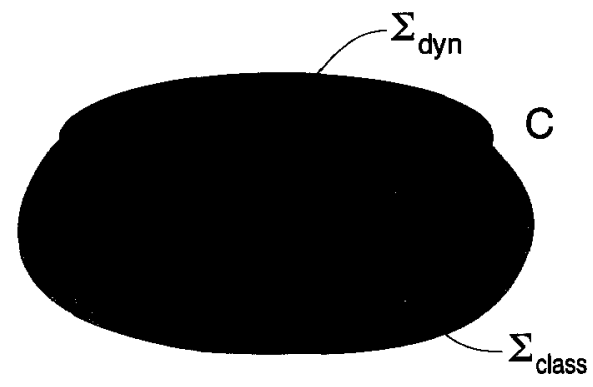

Fig. 17. The classical world sheet $\Sigma_{\text {class }}$ and the dynamical world sheet $\Sigma_{\text {dyn }}$ associated with the 't Hooft loop operator $B_{a}\left(C, \Sigma_{\text {class }}, x_{0}\right)$. 
If no stable $a$-string exists, then $B_{a}\left(C, \Sigma, x_{0}\right)$ might not create a stable string. If it does not, its expectation value will behave, for a large loop $C$, like

$$
\left\langle B_{a}\left(C, \Sigma, x_{0}\right)\right\rangle \sim \exp \left(-\kappa_{a}^{(\mathrm{ren})} A(\Sigma)\right) \exp \left(-m_{a}^{(\text {ren })} P(C)\right),
$$

where $P(C)$ is the perimeter of $C$. It may seem, then, that by measuring $\left\langle B_{a}\left(C, \Sigma, x_{0}\right)\right\rangle$, and determining whether it decays as in eq. (7.2) or as in eq. (7.3), we can find out whether $a$ is contained in the unbroken subgroup $H$ or not. However, there are subtleties. One problem is that there may be tradeoff between the dependence of $\left\langle B_{a}\left(C, \Sigma, x_{0}\right)\right\rangle$ on $A(\Sigma)$ and its dependence on $A(C)$. Eq. (7.3) will apply if the domain wall bounded by $\Sigma$ decays by nucleating an $a^{-1}$ string that completely cancels the flux of the classical string source on $\Sigma$. But it may be that $\kappa_{a}^{(\text {ren) }}$ can be reduced if the nucleated string only partially screens the flux of the source. (We saw an instance of this phenomenon in the $\mathbb{Z}_{6}$ example that was discussed in subsect. 4.2.) The advantage gained from reducing $\kappa^{(\mathrm{ren})}$ may more than compensate for the cost of a non-vanishing $\kappa^{(\mathrm{dyn})}$; then $B_{a}$ will decay as in eq. (7.2), even though $a \notin \mathrm{H}$.

Another complication can arise if the unbroken subgroup $\mathrm{H}$ is not a normal subgroup of $G$. For then a typical $G$ conjugacy class contains both elements that are in $\mathrm{H}$ and elements that are not in $\mathrm{H}$. Recall that $B_{a}\left(C, \Sigma, x_{0}\right)$ is actually averaged over the $\mathrm{G}$ conjugacy class that contains $a$. One particular H-class contained in this G-class will dominate the asymptotic behavior of $\left\langle B_{a}\left(C, \Sigma, x_{0}\right)\right\rangle$, and whether eq. (7.3) or eq. (7.2) applies depends on which class dominates.

We will return to the problem of finding a suitable order parameter, that can be used to identify $\mathrm{H}$, in sect. 9 .

\subsection{MIXING}

If the discrete gauge group $G$ is unbroken, then elements of $G$ in the same conjugacy class are associated with strings that transform into each other under the action of G. In the classical limit, these "group eigenstate" strings are degenerate energy eigenstates. (There is also a further degeneracy associated with "parity," which changes the orientation, and so transforms the $a$ string into the $a^{-1}$ string.) Quantum mechanically, these states mix with one another, and the degeneracy is lifted. The true energy eigenstates are "charge eigenstates" that transform according to irreducible representations of $\mathrm{G}$ (and parity) [21].

This mixing can be computed using the 't Hooft operator. We consider a correlation function in which an $a$-loop is created on $C_{1}$ and a $b$ loop is annihilated (or a $b^{-1}$ loop created) on $C_{2}$, where $b=g a g^{-1}$ for some $g \in \mathrm{G}$. It is crucial that the two strings be defined with respect to the same basepoint $x_{0}$. Otherwise, we would average $a$ and $b$ over the conjugacy class independently, and 


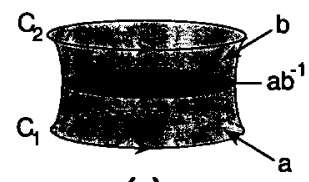

(a)

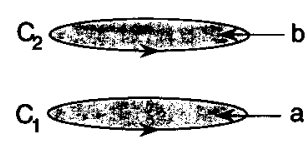

(b)

Fig. 18. Two contributions to the mixing of an $a$-string and a $b$-string. In (a), an $a b^{-1}$ string spontaneously nucleates, expands, meets the $a$-string, and converts it into a $b$-string. In (b), the $a$-string shrinks and annihilates, then the $b$-string nucleates and grows.

the correlation function would be dominated by the propagation of an $a$-string from $C_{1}$ to $C_{2}$, rather than the mixing of an $a$-string with a $b$-string.

Let $C_{1}$ and $C_{2}$ be two congruent loops, one directly above the other as in fig. 18. The separation between the loops is large compared to the correlation length of the theory, but small compared to the size of the loops. If the $a b^{-1}$ string is stable, then, in the weak coupling limit, the correlation function will be dominated by the configuration in fig. 18a. In this configuration, the world-sheets of the $a$ and $b$ strings join, and the loop at which they join is the boundary of the world sheet of an $a b^{-1}$ string. If this configuration dominates, then

$$
B_{a, b^{-1}}\left(C_{1}, \Sigma_{1}, C_{2}, \Sigma_{2}, x_{0}\right) \sim \exp \left(-\kappa_{a}^{(\mathrm{ren})} A\left(\Sigma_{1}\right)-\kappa_{b}^{(\mathrm{ren})} A\left(\Sigma_{2}\right)\right) \exp \left(-\kappa_{a b^{-}}^{(\mathrm{dyn})} A\left(C_{1}\right)\right)
$$

But if the $a b^{-1}$ string is unstable, and decays to a widely separated $a$-string and $b^{-1}$ string, then the configuration in fig. 18b will dominate. Here, the world-sheets of the dynamical strings are stretched tightly across $C_{1}$ and $C_{2}$. If this configuration dominates, then

$$
\begin{aligned}
& B_{a, b^{-1}}\left(C_{1}, \Sigma_{1}, C_{2}, \Sigma_{2}, x_{0}\right) \\
& \quad \sim \exp \left(-\kappa_{a}^{(\mathrm{ren})} A\left(\Sigma_{1}\right)-\kappa_{b}^{(\mathrm{ren})} A\left(\Sigma_{2}\right)\right) \exp \left(-\left(\kappa_{a}^{(\mathrm{dyn})}+\kappa_{b}^{(\mathrm{dyn})}\right) A\left(C_{1}\right)\right) .
\end{aligned}
$$

Thus, the mixing amplitude in the weak-coupling limit is either

$$
\exp \left(-S_{a, b}^{(\mathrm{mix})}\right) \sim \exp \left(-\left(\kappa_{a}^{(\mathrm{dyn})}+\kappa_{b}^{(\mathrm{dyn})}\right) A\left(C_{1}\right)\right)
$$

or

$$
\exp \left(-S_{a, b}^{(\text {mix })}\right) \sim \exp \left(-\kappa_{a b^{-1}}^{(\mathrm{dyn})} A\left(C_{1}\right)\right)
$$

whichever is larger. 


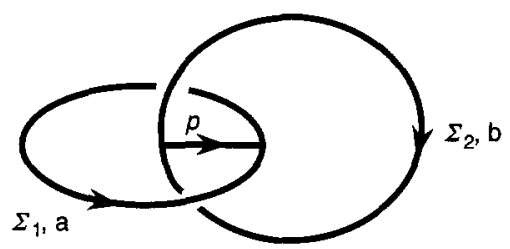

Fig. 19. Linked world lines $\Sigma_{1}$ and $\Sigma_{2}$ of a classical $a$-vortex and a classical $b$-vortex. If $a$ and $b$ do not commute, then the classical vortices must exchange a dynamical $a b a^{-1} b^{-1}$ vortex. The exchange occurs along the path $P$, the shortest path that connects the two world-lines. (Compare fig. 5.)

Actually, these are not the most general possibilities, for the $a b^{-1}$ string may be unstable, and may prefer to decay in some other channel. If the $a b^{-1}$ string decays to $n$ widely separated stable strings that carry flux $c_{1}, c_{2}, \ldots, c_{n}$, then we find

$$
\exp \left(-S_{a, b}^{(\operatorname{mix})}\right) \sim \exp \left(-\left(\sum_{i} \kappa_{c_{i}}^{(\mathrm{dyn})}\right) A\left(C_{1}\right)\right)
$$

These results were previously derived in ref. [21].

\section{Interactions}

\subsection{ENTANGLEMENT AND HOLONOMY INTERACTIONS}

The Aharonov-Bohm interaction between a classical string source and a classical charged source was studied in sect. 6. Correlation functions can also be used to study the holonomy interaction between two vortices (in $2+1$ dimensions), or two strings (in $3+1$ dimensions), and also the entanglement of strings.

In $2+1$ dimensions, two loops $\Sigma_{1}$ and $\Sigma_{2}$ can link, as shown in fig. 19 . Then the quantity $\left\langle F_{a, b}\left(\Sigma_{1}, \Sigma_{2}, x_{0}\right)\right\rangle$ probes what happens when one vortex source winds around another. As we noted in sect. 2 , there is a holonomy interaction between the vortices if $a$ and $b$ do not commute; both are conjugated by $a b[24,25]$. This means that the vortex world-lines cannot close (the vortex pairs cannot re-annihilate) unless there is an exchange of topological quantum numbers between the two vortices.

An $(a b) a(a b)^{-1}$ vortex becomes an $a$ vortex, and an $(a b) b(a b)^{-1}$ vortex becomes a $b$-vortex, if the flux $a b a^{-1} b^{-1}$ flows from the $b$-vortex to the $a$-vortex *

* Here we have used different conventions to assign group elements to the entangled vortex world-lines than the conventions used in subsect. 2.4 to assign group elements to entangled strings in three spatial dimensions. 
Therefore, the quantum numbers that must be exchanged are those of an $a b a^{-1} b^{-1}$ vortex. We thus find, in the weak-coupling limit,

$$
\begin{aligned}
& \left\langle F_{a, b}\left(\Sigma_{1}, \Sigma_{2}, x_{0}\right)\right\rangle \\
& \quad \sim \exp \left(-m_{a}^{(\text {ren })} P\left(\Sigma_{1}\right)-m_{b}^{(\text {ren })} P\left(\Sigma_{2}\right)\right) \exp \left(-m_{a b a-b^{-1}}^{\left(\text {dyn }{ }_{1}\right.} L\left(\Sigma_{1}, \Sigma_{2}\right)\right) .
\end{aligned}
$$

Here $m^{(\text {ren) }}$ is the renormalization of the mass of a classical vortex source, $m^{\text {(dyn) }}$ is the mass of a dynamical vortex, $P(\Sigma)$ is the perimeter of $\Sigma$, and $L\left(\Sigma_{1}, \Sigma_{2}\right)$ is the length of the shortest path that connects $\Sigma_{1}$ and $\Sigma_{2}$. (If the $a b a^{-1} b^{-1}$ vortex is unstable, then $m_{a b a^{-1} b^{-1}}^{(\mathrm{dyn}}$ is replaced by the sum of the masses of the vortices to which it decays.)

The leading behavior of weak-coupling perturbation theory on the lattice is found by identifying the configurations with the minimal number of excited plaquettes, as we described in sect. 6. To find the leading contribution to $\left\langle F_{a, b}\left(\Sigma_{1}, \Sigma_{2}, x_{0}\right)\right\rangle$, we choose surfaces $\Omega_{1}$ and $\Omega_{2}$ that are bounded by $\Sigma_{1}$ and $\Sigma_{2}$ respectively, and construct a configuration with $U_{l}=a$ on links in $\Omega_{1}^{*}$ and $U_{l}=b$ on links in $\Omega_{2}^{*}$. But where $\Omega_{1}$ and $\Omega_{2}$ intersect, there is a string of excited plaquettes with

$$
S_{\text {gauge }, P}^{(R)}=-\beta \chi^{(R)}\left(a b a^{-1} b^{-1}\right)+\text { c.c. }
$$

(See fig. 20.) By choosing $\Omega_{1}$ and $\Omega_{2}$ so that this string has minimal length, we obtain eq. (8.1).

(If the $a b a^{-1} b^{-1}$ vortex is unstable, then the dominant configuration in the weak-coupling limit is found by a slightly modified procedure. Either $\Sigma_{1}$ or $\Sigma_{2}$, or

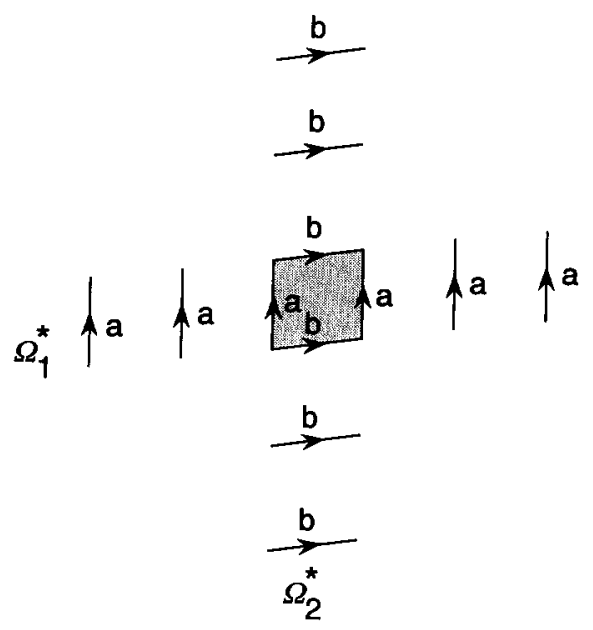

Fig. 20. A plaquette (shaded) contained in the intersection of $\Omega_{1}^{*}$ and $\Omega_{2}^{*}$. 


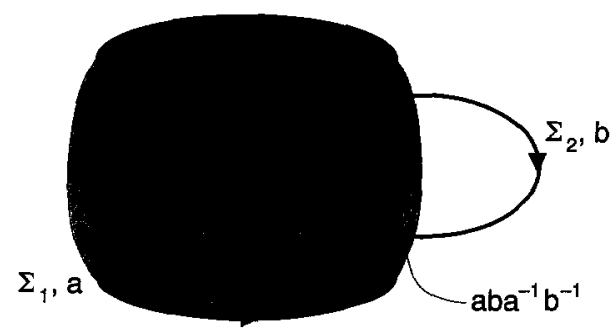

Fig. 21. World-sheets $\Sigma_{1}$ and $\Sigma_{2}$ of a classical $a$-string and classical $b$-string that intersect at two isolated points. (Only a slice through $\Sigma_{2}$ is shown.) The indicated shaded region is the world sheet of a dynamical $a b a^{-1} b^{-1}$ string that connects the two classical strings.

both, becomes the boundary of several surfaces $\Omega_{1}^{i}$, or $\Omega_{2}^{j}$. The link configuration is chosen so that $U_{l}=c_{i}$ on $\Omega_{1}^{* i}$ and $U_{l}=d_{j}$ on $\Omega_{2}^{* j}$, where $\Pi c_{i}=a$ and $\Pi d_{j}=b$. These surfaces intersect along several strings that connect the two world-lines; thus, the classical vortices exchange several separated dynamical vortices.)

In $3+1$ dimensions, strings labeled by non-commuting group elements become entangled when they cross, as we described in subsect. 2.4. String world-sheets in four dimensions generically intersect at isolated points. (A point of intersection is a type of "instanton.") Consider surfaces $\Sigma_{1}$ and $\Sigma_{2}$ that cross at two points, as shown in fig. 21. Because the strings entangle, an $a$ world sheet on $\Sigma_{1}$ and a $b$ world sheet on $\Sigma_{2}$ become joined by the world sheet of an $a b a^{-1} b^{-1}$ string. Thus, in the weak-coupling limit, we find

$$
\begin{aligned}
& \left\langle F_{a, b}\left(\Sigma_{1}, \Sigma_{2}, x_{0}\right)\right\rangle \\
& \quad \sim \exp \left(-\kappa_{a}^{(\mathrm{ren})} A\left(\Sigma_{1}\right)-\kappa_{b}^{(\mathrm{ren})} A\left(\Sigma_{2}\right)\right) \exp \left(-\kappa_{a b a}^{(\mathrm{dyn})_{b^{-1}}} A\left(\Sigma_{1}, \Sigma_{2}\right)\right),
\end{aligned}
$$

where $A\left(\Sigma_{1}, \Sigma_{2}\right)$ is the area of the minimal surface that joins $\Sigma_{1}$ and $\Sigma_{2}$. Again, this result is easily verified using weak-coupling perturbation theory on the lattice.

It is also instructive to consider $\left\langle F_{a, b}\left(\Sigma_{1}, \Sigma_{2}, x_{0}\right)\right\rangle$ where the surfaces $\Sigma_{1}$ and $\Sigma_{2}$ have the topology shown in fig. 22. Here $\Sigma_{1}$ is a torus that links once with the sphere $\Sigma_{2}$. As before, $\Omega_{1}$ and $\Omega_{2}$ unavoidably intersect along a surface of

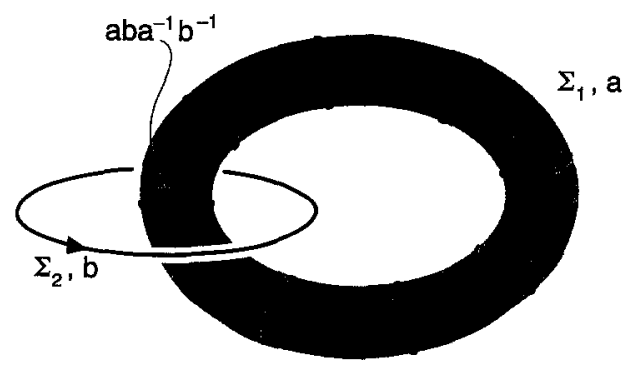

Fig. 22. World-sheets $\Sigma_{1}$ and $\Sigma_{2}$ of a classical $a$-string and a classical $b$-string. (Only a slice through $\Sigma_{2}$ is shown.) $\Sigma_{1}$ is a torus that links with $\Sigma_{2}$. The shaded region is the world-sheet of a dynamical $a b a^{-1} b^{-1}$ string. 
frustrated plaquettes; we find the leading behavior of $\left\langle F_{a, b}\left(\Sigma_{1}, \Sigma_{2}, x_{0}\right)\right\rangle$ by choosing $\Omega_{1}$ and $\Omega_{2}$ so that this intersection has minimal area. The result is again eq. (8.3). But now, if the width of the torus is small compared to the size of the sphere, $A\left(\Sigma_{1}, \Sigma_{2}\right)$ is the area of the minimal slice through the torus.

To interpret this result, we recall the observation in subsect. 2.3 , that when a loop of $a$-string winds around a loop of $b$-string, it becomes a loop of $b a b^{-1}$ string. We may regard fig. 22 as a depiction of a process in space-time, in which loops of $a$ and $a^{-1}$ string are produced, the $a$-loop winds around the $b$-string, and the $a$ and $a^{-1}$ strings then annihilate. But because of the Aharonov-Bohm interaction between the strings, this process is disallowed; an $a^{-1}$ loop cannot annihilate a $b a b^{-1}$ loop. Therefore, $\left\langle F_{a, b}\left(\Sigma_{1}, \Sigma_{2}, x_{0}\right)\right\rangle$ is suppressed by the amplitude for the $b a b^{-1}$ string to become, via quantum tunneling, an $a$-string. Indeed, comparing with eq. (7.7), we see that

$$
\exp \left(-S_{a, b a b^{-1}}^{(\operatorname{mix})}\right) \sim \exp \left(-\kappa_{a b a^{-1} b^{-1}}^{(\mathrm{dyn})} A^{(\min )}\left(\Sigma_{1}\right)\right)
$$

is the suppression factor in eq. (8.3).

\subsection{CHESHIRE CHARGE AND THE BORROMEAN RINGS}

As discussed in subsect. 2.2, it is an inevitable consequence of the non-abelian Aharonov-Bohm phenomenon that a loop of string (or a pair of vortices) can carry charge, and can exchange charge with other charged objects [5,12,13]. Let us see how this property is reflected in the behavior of the correlation functions of our string operators [30].

Consider the following process (in $2+1$ dimensions), depicted in fig. 23. First, a pair consisting of an $a$-vortex and its anti-vortex spontaneously appears; the total

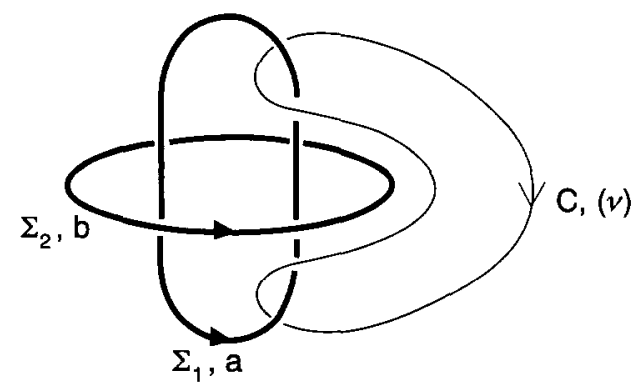

Fig. 23. The Borromean rings. $\Sigma_{1}$ is the world-line of an $a$-vortex, $\Sigma_{2}$ is the world-line of a $b$-vortex, and $C$ is the world-line of a charged particle that transforms as the representation $(\nu)$. The charged particle transfers charge to the $a$ vortex-antivortex pair, and the charge is subsequently detected via the Aharonov-Bohm interaction of the pair with the $b$-vortex. 


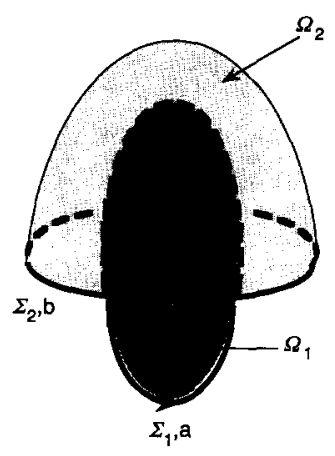

Fig. 24. Non-intersecting surfaces $\Omega_{1}$ and $\Omega_{2}$ that are bounded by the vortex world-lines $\Sigma_{1}$ and $\Sigma_{2}$.

charge of the pair is trivial. Then a charged particle in the representation $(\nu)$ winds counterclockwise around the $a$-vortex; thus, charge is transferred to the vortex pair, as described in subsect. 2.2. Next, a $b$-vortex winds around the (charged) vortex pair, acquiring an Aharonov-Bohm phase that is sensitive to the charge of the pair. Then the $(\nu)$ particle winds clockwise around the $a$-vortex, discharging the pair. Finally, the $a$-vortex and anti-vortex re-unite and annihilate.

If the vortices and charged particle are treated as classical sources, this process is captured by the correlation function $\left\langle F_{a, b}\left(\Sigma_{1}, \Sigma_{2}, x_{0}\right) W^{(\nu)}(C)\right\rangle$, where the three loops $\Sigma_{1}, \Sigma_{2}$ and $C$ are configured as in fig. 23. This is a topologically non-trivial joining of three loops known as the "Borromean rings;" no two loops are linked, yet the loops cannot be separated without crossing. Because this correlation function has the space-time interpretation described above, we may anticipate that, when the three loops are joined but far apart, $\left\langle F_{a, b}\left(\Sigma_{1}, \Sigma_{2}, x_{0}\right) W^{(\nu)}(C)\right\rangle$ differs from its value for large unjoined loops by a topological multiplicative factor - the Aharonov-Bohm phase acquired by the $b$-vortex that winds around the charged vortex pair.

To calculate $A_{a, b}^{(\nu)}\left(\Sigma_{1}, \Sigma_{2}, x_{0} ; C\right)$, we proceed, as usual, by finding the configuration of the link variables such that, when $F_{a, b}$ is inserted, there are no frustrated plaquettes. We pick surfaces $\Omega_{1}$, bounded by $\Sigma_{1}$, and $\Omega_{2}$, bounded by $\Sigma_{2}$, that do not intersect; such surfaces are shown in fig. 24 . We then choose (up to a gauge transformation)

$$
\begin{aligned}
& U_{l}=a, \quad l \in \Omega_{1}^{*}, \\
& U_{l}=b, \quad l \in \Omega_{2}^{*}, \\
& U_{l}=e, \quad l \notin \Omega_{1}^{*} \cup \Omega_{2}^{*} .
\end{aligned}
$$


Now we compute $W^{(v)}(C)$ in this configuration. As is clear in fig. 24, the loop $C$ crosses first $\Omega_{1}$ in a positive sense, then $\Omega_{2}$ in a negative sense, then $\Omega_{1}$ in a negative sense, and finally $\Omega_{2}$ in a positive sense, before closing. The corresponding path-ordered exponential is $b a^{-1} b^{-1} a$, and taking a trace yields [30]

$$
\lim \left\langle A_{a, b}^{(\nu)}\left(\Sigma_{1}, \Sigma_{2}, x_{0} ; C\right)\right\rangle \sim \frac{1}{n_{\nu}} \chi^{(\nu)}\left(a b a^{-1} b^{-1}\right) .
$$

We should verify that the factor (8.6) can be interpreted as the Aharonov-Bohm phase acquired by a $b$-vortex that winds around a vortex pair with Cheshire charge. The interpretation is easiest if we explicitly average $a$ over the representatives of the class to which it belongs. If this averaging is not performed, then there is a correlation between the choice of the class representative that is used to measure the charge, and the choice of the class representative for the string that is being measured; this correlation makes the interpretation of the measurement more complicated. Of course, averaging $a$ and $b$ over class representatives independently is equivalent to defining $a$ and $b$ with reference to distinct basepoints; we have

$$
\lim \frac{\left\langle F_{a}\left(\Sigma_{1}, x_{0}\right) F_{b}\left(\Sigma_{2}, y_{0}\right) W^{(\nu)}(C)\right\rangle}{\left\langle F_{a}\left(\Sigma_{1}, x_{0}\right)\right\rangle\left\langle F_{b}\left(\Sigma_{2}, y_{0}\right)\right\rangle\left\langle W^{(\nu)}(C)\right\rangle}=\frac{1}{n_{\mathrm{G}}} \sum_{g \in \mathrm{G}} \frac{1}{n_{\nu}} \chi^{(\nu)}\left(\mathrm{gag}^{-1} b g a^{-1} g^{-1} b^{-1}\right) .
$$

We recall from the discussion in subsect. 2.2 that, when a particle in representation $(\nu)$ winds around one of the vortices of a pair that is initially uncharged, the final state of the vortex pair is a superposition of states with various values of the charge. Eq. (8.7) gives the expectation value of the Aharonov-Bohm phase acquired by a $b$ vortex that winds around the charged pair. If $p_{\mu}$ is the probability that the vortex pair transforms as the irreducible representation $\mu$, then we have

$$
\sum_{\mu} p_{\mu} \frac{1}{n_{\mu}} \chi^{(\mu)}(b)=\frac{1}{n_{\mathrm{G}}} \sum_{g \in \mathrm{G}} \frac{1}{n_{\nu}} \chi^{(\nu)}\left(g a g^{-1} b g a^{-1} g^{-1} b^{-1}\right) .
$$

Indeed, eq. (8.8) can be verified directly, with $p_{\mu}$ given by eqs. (2.11) and (2.10). Details will be presented elsewhere [30].

The above discussion can be applied, with hardly any modification, to the case of charged string loops, in $3+1$ dimensions. There is an analog of the Borromean ring configuration, in which two disjoint closed surfaces are joined by a closed loop, although the loop is not linked with either surface. Eq. (8.7) holds, if $\Sigma_{1,2}$ are surfaces, and $C$ is a loop, in this configuration. 


\section{Gauge-Higgs systems}

\subsection{WEAK COUPLING}

In this section, we will consider models with Higgs fields coupled to gauge fields. We wish to investigate the screening of gauge charges - and of AharonovBohm interactions - due to the Higgs mechanism.

For a gauge system with (discrete) gauge group $\mathrm{G}$, we introduce a Higgs field $\phi_{i}$, defined on sites, that takes values in G. We then include in the action of the model the term

$$
S_{\mathrm{Higgs}}=-\sum_{\mu} \gamma_{\mu} \sum_{l}\left(\chi^{(\mu)}\left(\left(\phi^{-1} U \phi\right)_{l}\right)+\text { c.c. }\right)
$$

where the sum runs over all irreducible representations $(\mu)$ of G. (Compare eq. (4.3).) Suppose that some of the $\gamma_{\mu}$ are large ( $\gg 1$ ), while all others are small (or zero). Then, we can analyze the behavior of this model using perturbative methods.

In the weak-coupling limit $\gamma_{\mu} \rightarrow \infty, \phi^{-1} U \phi$ becomes restricted to the kernel of the representation $(\mu)$ at each link, and so $U_{P}$ is also "frozen" to the kernel at each plaquette. Thus, G becomes "spontaneously broken" to the subgroup $\mathrm{H}=$ $\operatorname{Ker}\left(D^{(\nu)}\right)$. By choosing several of the $\gamma_{\mu}$ to be large, we can break $\mathrm{G}$ to the kernel of a reducible representation. Indeed, breakdown to any normal subgroup of $\mathrm{G}$ can be achieved in this way, since every normal subgroup is the kernel of some representation.

Of course, we know from the usual continuum weak-coupling analysis that more general patterns of symmetry breakdown are possible. In that analysis, the Higgs field resides in the vector space on which a representation of $G$ acts, and, in principle, the unbroken subgroup could be the stability group of any vector in this space. In the model with $S_{\text {Higgs }}$ given by (9.1), these more general patterns are not obtained when all of the Higgs couplings either vanish or are very large. They might, of course, be obtained at intermediate coupling. After suitable block-spin transformations are performed, the effective theory that describes the infrared behavior of the model would be a "continuum" theory for which the usual analysis could apply.

Anyway, with the breaking of $\mathrm{G}$ to a normal subgroup $\mathrm{H}$ implemented as described above, we can proceed to calculate the ABOP $A_{a}^{(\nu)}\left(\Sigma, x_{0} ; C\right)$ in weakcoupling perturbation theory, and so probe the fate of the Aharonov-Bohm interaction in the Higgs model. The calculation gives the expected results. But there is one difficulty.

The problem is that the irreducible representation $(\nu)$ of $\mathrm{G}$ is typically a reducible representation of the subgroup $H$. In general, just one of the irreducible 
representations of $\mathrm{H}$ that is contained in $(\nu)$ will dominate the asymptotic behavior of $W^{(\nu)}(C)$ when the loop $C$ is large. So it is the Aharonov-Bohm interaction of this $\mathrm{H}$ representation with an $a$-string that is probed by $A_{a}^{(\nu)}$. In fact, if $(\nu)$ contains the trivial $\mathrm{H}$ representation, then this will dominate at weak coupling, and $\left\langle A_{a}^{(\nu)}\right\rangle$ will behave trivially even though $(\nu)$ may contain other $\mathrm{H}$-representations that $d o$ have Aharonov-Bohm interactions with the string.

We have encountered a general problem with the interpretation of $\left\langle A_{a}^{(\nu)}\right\rangle$ that arises whenever $\mathrm{G}$ is non-abelian and is partially broken. If $\left\langle A_{a}^{(\nu)}\right\rangle$ behaves non-trivially for some choice of $a$, then we know that an unscreened charge must dominate $W^{(\nu)}$; by varying $a$, we can obtain information about the representation according to which this charge transforms. But if $\left\langle A_{a}^{(\nu)}\right\rangle$ behaves trivially for all $a$, we know only that $W^{(\nu)}$ is dominated by a screened charge. We have no a priori knowledge of how this charge transforms, or of how other H-representations contained in $(\nu)$ interact with strings. This problem complicates the task of inferring $\mathrm{H}$ from the behavior of our correlation functions.

\subsection{STRING STABILITY}

We noted in subsect. 7.1 that, if $G$ is a discrete group, then the existence (or not) of a stable string with flux $a \in \mathrm{G}$ provides a criterion for determining whether $a$ is contained in the unbroken group $H$. It may seem, then, that the operator $B_{a}\left(C, \Sigma, x_{0}\right)$, which creates an $a$-string, can be used to probe the realization of the $\mathrm{G}$ gauge symmetry. But there are two problems.

First, when inserted in a Green function with gauge-invariant operators, $B_{a}$ is actually averaged over the $\mathrm{G}$ conjugacy class to which $a$ belongs. This class may contain some elements that are in the unbroken group $\mathrm{H}$ and some that are not, which complicates the interpretation of $\left\langle B_{a}\right\rangle$.

Second, even if no element of the class that contains $a$ is in $\mathrm{H}, B_{a}\left(C, \Sigma, x_{0}\right)$ may nevertheless create a stable string. This can happen, as we saw in subsect. 4.2, due to the competition between the renormalization of the tension of the classical string that propagates on $\Sigma$ and the tension of the dynamical string whose world sheet is bounded by $C$.

Let us ignore the second problem for the moment, and address the first. We first note that the situation is relatively simple if $H$ is a normal subgroup of $G$. In that case, a given $\mathrm{G}$ conjugacy class is either entirely contained in $\mathrm{H}$ or is disjoint from $\mathrm{H}$. By studying $B_{a}$ for various class representatives, we can determine which classes are contained in $\mathrm{H}$, and so reconstruct $\mathrm{H}$.

The general case is somewhat more complicated, and $B_{a}$ by itself is not sufficient to completely determine the unbroken subgroup $\mathrm{H}$. Instead, one way to proceed is the following: If $\mathrm{G}=\left\{a_{1}, a_{2}, \ldots, a_{n_{\mathrm{G}}}\right\}$ is a finite group of order $n_{\mathrm{G}}$, consider

$$
\left\langle B_{a_{1}, a_{2}, \ldots, a_{n_{G}}}\left(C_{1}, \Sigma_{1}, C_{2}, \Sigma_{2}, \ldots, C_{n_{\mathrm{G}}}, \Sigma_{n_{\mathrm{G}}}, x_{0}\right)\right\rangle
$$


in which all group elements appear. Choose the loops to be large and widely separated, with their sizes ordered according to

$$
A\left(C_{1}\right) \gg A\left(C_{2}\right) \gg \ldots \gg A\left(C_{n_{\mathrm{G}}}\right)
$$

Now $B_{a_{1}, a_{2}, \ldots}$ is effectively averaged over gauge transformations at the basepoint, and so may be replaced by

$$
\frac{1}{n_{\mathrm{G}}} \sum_{g \in \mathrm{G}} B_{g a_{1} g^{-1}, g a_{2} g^{-1}, \ldots, g a_{n_{\mathrm{G}}} g^{-1}}\left(C_{1}, \Sigma_{1}, C_{2}, \Sigma_{2}, \ldots, C_{n_{\mathrm{G}}}, \Sigma_{n_{\mathrm{G}}}, x_{0}\right)
$$

The expectation value is dominated by the configurations that minimize

$$
\kappa_{g a_{1} g^{-1}}^{(\mathrm{ren})} A\left(\Sigma_{1}\right)+\kappa_{g a_{1} g^{-1}}^{(\mathrm{dyn})} A\left(C_{1}\right)
$$

(where $\kappa_{a}^{(\mathrm{dyn})}=0$ for $a \notin \mathrm{H}$ ). This condition may not determine $g$ uniquely. Among those $g$ that minimize eq. (9.5), the dominant configurations are such that $\kappa_{a_{2}}^{(\text {ren })} A\left(\Sigma_{2}\right)+\kappa_{a_{2}}^{(\mathrm{dyn})} A\left(C_{2}\right)$ is also minimized. And so on. Now by varying $A(C)$ and $A(\Sigma)$ independently, we find the group elements $a$ for which $\kappa_{g a g}^{\left(\text {dyn }_{1}\right.}=0$. We thus determine the unbroken subgroup up to one overall conjugacy $\mathrm{H} \rightarrow g \mathrm{Hg}^{-1}$. This ambiguity is expected; it corresponds to the freedom to change the embedding of $\mathrm{H}$ in $\mathrm{G}$ by performing a gauge transformation. (Of course, the calculation of eq. (9.2) involves an average with respect to this embedding.)

In passing, we have found the tension of all of the stable strings associated with the various elements of $H$.

If $\mathrm{G}$ is abelian, as in the discussion in subsect. 4.2 , then we can overcome the second problem (that $B_{a}$ may create a stable string even for $a \notin \mathrm{G}$ ) easily enough. By measuring $A_{a}^{(\nu)}$, we can determine the Aharonov-Bohm interactions of the string created by $B_{a}$, and so identify the flux of the string as belonging to $\mathrm{H}$. If $\mathrm{G}$ is non-abelian, though, life is more complicated. For as we noted above, we have no a priori knowledge of what $\mathrm{H}$-representation dominates the asymptotic decay of $W^{(\nu)}$. While it seems altogether physically reasonable that the stability and Aharonov-Bohm interactions of strings can be used to identify an unbroken gauge group $\mathrm{H}$, it is not so easily to specify how this should be done with gauge-invariant correlation functions.

\subsection{THE VOOP}

- Another promising probe of charge screening in a gauge theory is the "vacuum overlap order parameter" (VOOP) proposed by Fredenhagen and Marcu [17,18,20]. Let us compare and contrast the VOOP with the Aharonov-Bohm order parameter that has been discussed in this paper. 


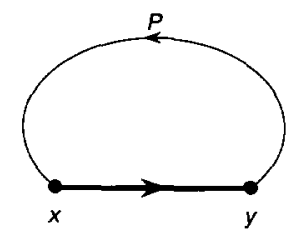

Fig. 25. The path $P$ from $y$ to $x$ that is used to construct the gauge-invariant correlation function $K^{(\mu)}(x, y, P)$. A classical charged particle propagates along $P$, and a dynamical charged particle propagates from $x$ to $y$.

Suppose that an $\mathrm{H}$ gauge theory contains a matter field $\Phi^{(\mu)}$ that transforms as the irreducible representation $(\mu)$ of $\mathrm{H}$. If the local H-symmetry is unbroken, and the representation $(\mu)$ is not confined or screened, then (loosely speaking) the field $\Phi^{\dagger}$ should create a stable particle. We can express this in gauge-invariant language. If $x$ and $y$ are distantly separated points, and $P_{x, y}$ is a path from $y$ to $x$, consider the non-local gauge-invariant operator

$$
K^{(\mu)}\left(x, y, P_{x, y}\right)=\Phi_{x}^{(\mu) \dagger} D^{(\mu)}\left(\prod_{l \in P_{x, y}} U_{l}\right) \Phi_{y}^{(\mu)} .
$$

If $\Phi^{(\mu)^{\dagger}}$ creates a free charge, then this charge must propagate between $x$ and $y$, as in fig. 25. Thus, we have

$$
\left\langle K^{(\mu)}(x, y, P)\right\rangle \sim \exp \left(-M_{\mathrm{ren}}^{(\mu)} L(P)\right) \exp \left(-M_{\mathrm{dyn}}^{(\mu)}|x-y|\right),
$$

where $M_{\mathrm{dyn}}^{(\mu)}$ is the mass of the stable particle created by $\Phi^{(\mu) \dagger}$, and $M_{\mathrm{ren}}^{(\mu)}$ is the renormalization of the mass of the classical source propagating along $P$. (Here, $L(P)$ is the length of $P$, and $|x-y|$ is the distance from $x$ to $y$.) Since $M_{\text {ren }}^{(\mu)}$ can be determined independently by measuring $\left\langle W^{(\mu)}(C)\right\rangle$ (or by varying $L(P)$ with $|x-y|$ fixed), eq. (9.7) can be used to find $M_{\mathrm{dyn}}^{(\mu)}$. But if the representation $(\mu)$ is screened (or confined), then $\left\langle K^{(\mu)}(x, y, P)\right\rangle$ will become independent of $|x-y|$ for large separation; in effect, we have $K_{\mathrm{dyn}}^{(\mu)}=0$, with $M_{\mathrm{dyn}}^{(\mu)}$ defined by eq. (9.7). Thus, Fredenhagen and Marcu suggested that $M_{\mathrm{dyn}}^{(\mu)}>0$ if and only if $\Phi^{(\mu) \dagger}$ creates a free charge.

This construction is obviously closely similar to our discussion in subsect. 7.1 of string stability, and it suffers from related problems. Suppose that, in a model with gauge group $\mathrm{G}$, spontaneous breakdown to a subgroup $\mathbf{H}$ occurs. Then, an irreducible representation $(\mu)$ of $\mathrm{G}$ contains various irreducible representations of H. It may be that among these representations are some that can exist as free charges, and others that are screened. We might expect, then, that the screened charges dominate $\left\langle K^{(\mu)}\right\rangle$, so that $M_{\mathrm{dyn}}^{(\mu)}=0$ even though $(\mu)$ contains some unscreened representations of $H$. Even this is not clear; because of the competition between $M_{\mathrm{dyn}}$ and $M_{\text {ren }}$ in eq. (9.7), the free charges may actually dominate. 
Because of these difficulties, it is not at all easy, in general, to identify the unbroken gauge group $H$ based on the behavior of $\left\langle K^{(\mu)}(x, y, P)\right\rangle$.

\subsection{ORDER PARAMETERS: SOME CONCLUDING REMARKS}

The existence of stable cosmic strings (or vortices), and of Aharonov-Bohm interactions between strings and free charges, can be used to identify and classify the various phases of a gauge theory. Yet, because of the problems discussed above, it proves difficult to formulate a general procedure that unambiguously specifies the realization of the gauge symmetry, e.g. the "unbroken" subgroup. This is surprising (to us), but we are reluctant to attach any fundamental significance to it.

In fact, though, just because of these problems, the "phase structure" of certain gauge theories may be richer than one might naively expect. For example, if the gauge group G is "spontaneously broken" to $\mathrm{H}$, then, as we have remarked, just one of the irreducible $H$ representations contained in the $G$ representation $(\nu)$ will dominate the asymptotic behavior of $\left\langle W^{(\nu)}(C)\right\rangle$. On some surface in the parameter space of the theory, a "crossover" may occur, where this H-representation changes. Thus, the order parameter $A_{a}^{(\nu)}$ might be non-analytic on this surface. However, no change in symmetry occurs on this surface, and it should not be regarded as a genuine phase boundary.

For most of the discussion in this paper, we have taken the gauge group $G$ to be a discrete group. There is no obstacle, however, to generalizing our results to the case where $G$ is a continuous group.

\section{Electric flux tubes and dynamical monopoles}

In this paper, we have systematically discussed the Aharonov-Bohm interactions between magnetic flux tubes and electric charges, which can occur in the Higgs phase of a gauge theory. Central to the discussion has been the effect of quantum-mechanical electric charge fluctuations on the interaction.

There is another type of Aharonov-Bohm interaction, which can occur in the confining phase of a gauge theory-the interaction between an electric flux tube and a magnetic charge. The existence of such interactions has been noted previously $[5,9,22,23]$ as have the implications concerning the "magnetic hair" carried by black holes [5,9]. But the effects of quantum-mechanical magnetic charge fluctuations on this incarnation of the Aharonov-Bohm phenomenon have not been analyzed before.

In this section, we will develop a quantum field-theoretic treatment of electric flux tubes in a confining gauge theory that contains dynamical magnetic monopoles, and will investigate the interactions of flux tubes with monopoles. This treatment, 
of course, will be closely similar to our theory of magnetic flux tubes, in a Higgs phase.

First, we will describe (following closely the work of Srednicki and Susskind [23] - how dynamical magnetic monopoles are introduced into a lattice gauge theory. Then we will construct a generalized Wilson loop operator that creates an electric flux tube that has Aharonov-Bohm interactions with the monopoles. By studying the properties of the correlation functions of this operator, we will investigate the effect of magnetic charge fluctuations on the Aharonov-Bohm effect.

\subsection{MAGNETIC MONOPOLES ON THE LATTICE}

For definiteness, we will consider a gauge theory with gauge group $\mathrm{SU}(N)$. A pure SU( $N)$ gauge theory (no matter), or a theory with the matter fields transforming trivially under the center $\mathbb{Z}_{N}$ of $\mathrm{SU}(N)$, admits magnetic monopoles with $\mathbb{Z}_{N}$ magnetic charges. (Fields that transform under $\mathbb{Z}_{N}$ would be able to see the Dirac string of such a monopole; that is, the monopole with minimal $\mathbb{Z}_{N}$ charge would not satisfy the Dirac quantization condition.) If $S U(N) / \mathbb{Z}_{N}$ is actually the unbroken gauge symmetry of an underlying theory with simply connected gauge group $\mathrm{G} \supset \mathrm{SU}(N) / \mathbb{Z}_{N}$, where $\mathrm{G}$ is broken via the Higgs mechanism, then such monopoles arise as topological solitons. For example, there are models with $\mathrm{G}=\operatorname{Spin}\left(N^{2}-1\right)$ broken to $\operatorname{SU}(N) / \mathbb{Z}_{N}$ that contain $\mathbb{Z}_{N}$ monopoles.

The (usual) 't Hooft loop operator $B(C)$ inserts a world line of a $\mathbb{Z}_{N}$ monopole along the closed loop $C$. But the monopole introduced by an 't Hooft loop is a classical source, not a dynamical object. We wish to elevate the monopoles to the status of dynamical degrees of freedom, and introduce a coupling constant that controls the effects of virtual monopoles.

It is most convenient to choose the dynamical variable to be a "Dirac string field" that is summed over in the path integral. On the lattice, this field associates with each plaquette $P$ a quantity $\eta_{P} \in \mathbb{Z}_{N}$, which may be regarded as the $\mathbb{Z}_{N}$ magnetic flux carried by a Dirac string that pierces that plaquette. If the total magnetic flux entering a cube of the lattice is nontrivial, then a magnetic monopole resides in that cube. Of course, since the Dirac strings themselves must be unobservable, this theory should respect, as well as the usual $\operatorname{SU}(N)$ local symmetry, an additional $\mathbb{Z}_{N}$ local symmetry that deforms the Dirac strings (without, of course, changing where the monopoles are).

The construction of this theory was described by Srednicki and Susskind [23]. In the absence of matter fields, it has the action

$$
S=-\beta \sum_{\text {plaquettes } P} \operatorname{tr}\left(\eta_{P} U_{P}\right)-\lambda \sum_{\text {cubes } c} \eta_{c}+\text { c.c. }
$$


where

$$
\eta_{c}=\prod_{P \in c} \eta_{P}
$$

is the product of the six $\eta_{P}$ associated with the oriented faces of the cube $c$; in other words, $\eta_{c}$ is the $\mathbb{Z}_{N}$ magnetic charge inside $c$.

The extra $\mathbb{Z}_{N}$ local symmetry respected by this theory is defined on links; it acts according to

$$
\begin{aligned}
n: U_{l} & \rightarrow \mathrm{e}^{2 \pi i n / N} U_{l}, \\
\eta_{P} & \rightarrow \mathrm{e}^{-2 \pi i n / N} \eta_{P}, \quad l \in P,
\end{aligned}
$$

where $n=0,1,2, \ldots, N-1$. This transformation moves the Dirac strings without changing the magnetic charge $\eta_{c}$ or the magnetic flux $\eta_{P} U_{P}$ that appears in the gauge field plaquette action. (The quantity $U_{P}$ is not invariant under the extra local symmetry, and so is unphysical; it can be interpreted as a fictitious magnetic flux that includes a contribution from the (unobservable) Dirac string that crosses P.)

The coupling constant $\lambda$ controls the strength of the effects of virtual monopoles. For $\lambda \ll 1$, magnetic charge fluctuations occur copiously, but for $\lambda \gg 1$, magnetic charge fluctuations are strongly suppressed. In the limit $\lambda \rightarrow \infty$, the monopoles freeze out, and eq. (10.1) becomes the usual Wilson action.

Matter fields can be coupled to the gauge theory in the usual way. For example if $\phi_{i} \in \mathrm{SU}(N)$ is defined on sites (labeled by $i$ ), we may define

$$
S_{\text {matter }}=-\gamma \sum_{\text {links }} \chi^{(R)}\left(\left(\phi^{-1} U \phi\right)_{l}\right)+\text { c.c.. }
$$

This is invariant under the local symmetry eq. (10.3) only if $\mathbb{Z}_{N}$ is contained in the kernel of the representation $(R)$. Of course, this is just the Dirac quantization condition - the matter fields must be chosen so that the string of a monopole is invisible. We may introduce matter fields that are only invariant under some subgroup of $\mathbb{Z}_{N}$, but then we must restrict the $\eta_{P}$ to take values in that subgroup.

\subsection{WILSON LOOP OPERATOR}

Now we want to define a Wilson loop operator $W^{(\nu)}(C)$ that creates an electric flux tube on the loop $C$. But the usual construction

$$
W^{(\nu)}(C)=\frac{1}{n_{\nu}} \chi^{(\nu)}\left(\prod_{l \in C} U_{l}\right)
$$


is not invariant under the local symmetry eq. (10.3) unless $\mathbb{Z}_{N}$ is contained in the kernel of the representation $(\nu)$. The operator $W^{(\nu)}(C)$ is physically sensible, then, only if the string created by it has no Aharonov-Bohm interaction with a $\mathbb{Z}_{N}$ monopole. In fact, in the SU(N) theory defined by eq. (10.1), this operator does not create a stable flux tube at all - even at strong coupling $(\beta \ll 1)$, glue fluctuations cause the string to break.

An operator that creates a stable electric flux tube that does have AharonovBohm interactions with monopoles cannot depend on the loop $C$ alone; it must also depend on a surface $\Sigma$ that is bounded by $C$. After our discussion of the 't Hooft operator in subsect. 5.3, this comes as no surprise. A (naive) Wilson loop operator, in the presence of monopoles, is a multi-valued object, for it acquires a non-trivial Aharonov-Bohm phase when the loop winds around a magnetic charge. To construct a single-valued object, we introduce a branch cut on the surface $\Sigma$, so that the operator jumps discontinuously when a monopole crosses $\Sigma$. We augment the naive Wilson loop operator, then, by a factor that counts the total magnetic flux of the Dirac strings that cross $\Sigma$, obtaining

$$
W^{(\nu)}(C, \Sigma) \equiv \frac{1}{n_{\nu}} \chi^{(\nu)}\left(\left(\prod_{l \in C} U_{l}\right)\left(\prod_{P \in \Sigma} \eta_{P}\right)\right) .
$$

This operator is invariant under eq. (10.3), for any representation $(\nu)$ of $\operatorname{SU}(N)$, and of course it reduces to $W^{(\nu)}(C)$ if $(\nu)$ represents $\mathbb{Z}_{N}$ trivially.

We may also consider the degenerate case in which the loop $C$ shrinks to a point. If $(\nu)$ represents $\mathbb{Z}_{N}$ faithfully, then the operator

$$
G^{(\nu)}(\Sigma)=\frac{1}{n_{\nu}} \chi^{(\nu)}\left(\prod_{P \in \Sigma} \eta_{P}\right)
$$

inserts the world-sheet of a tube with minimal $\mathbb{Z}_{N}$ electric flux, as a classical source, on the surface $\Sigma$. (Of course, if $(\nu)$ represents $\mathbb{Z}_{N}$ trivially, then the flux tube is invisible, and the operator is trivial.) If we think of the surface $\Sigma$ as lying in a time slice, then $G(\Sigma)$ has another interpretation; it is a magnetic charge operator that measures the total $\mathbb{Z}_{N}$ magnetic flux through $\Sigma$. Obviously, $G(\Sigma)$ is the magnetic analogy of the operator that we called $F(\Sigma)$ in sect. 4 .

Note also that if matter is introduced as in eq. (10.4), an operator can be constructed that creates a separated particle-antiparticle pair; it is

$$
\chi^{(R)}\left(\phi_{i}^{-1}\left(\prod_{l \in P_{i, j}} U_{l}\right) \phi_{j}\right)
$$

where $P_{i, j}$ is a path from the site $i$ to the site $j$. This operator is invariant under the local symmetry, if $(R)$ represents $\mathbb{Z}_{N}$ trivially. 


\section{3. 't HOOFT LOOP OPERATOR}

We can also construct an 't Hooft loop operator $B_{n}(C)$; it inserts on the world line $C$ a classical monopole source with $\mathbb{Z}_{N}$ magnetic charge $n=1,2, \ldots, N-1$. Alternatively, we may interpret $B_{n}(C)$, acting in a time slice, as an operator that creates a loop of magnetic flux tube on $C$.

The construction of this 't Hooft operator may be carried out in much the same way as in a gauge theory without dynamical monopoles. We regard $C$ as a closed loop composed of links of the dual lattice, and we chose an arbitrary surface $\Sigma$ (composed of plaquettes of the dual lattice) whose boundary is $C$. Dual to the plaquettes of $\Sigma$ is a set $\Sigma^{*}$ of plaquettes of the original lattice. Now, if there are no dynamical monopoles, we regard the $\eta_{P}$ as classical (non-fluctuating) variables. Then, to evaluate a Green function with an insertion of $B_{n}(C)$, we modify the plaquette action on the plaquettes in $\Sigma^{*}$, by choosing

$$
\begin{array}{ll}
\eta_{P}=\mathrm{e}^{2 \pi i n / N} & P \in \Sigma^{*} . \\
\eta_{P}=1, & P \notin \Sigma^{*} .
\end{array}
$$

When there are dynamical monopoles, however, and the $\eta_{P}$ are in the configuration eq. (10.9), the cubes that are dual to the links of $C$ are frustrated. Thus, this configuration represents a dynamical monopole propagating on the world line $C$. Since we want the operator $B_{n}(C)$ to introduce a classical monopole source, we should modify the cube action so that the configuration eq. (10.9) does not frustrate any cubes. Thus, we evaluate a Green function with an insertion of $B_{n}(C)$ by changing the cube action according to

$$
\eta_{c} \rightarrow \mathrm{e}^{2 \pi i n / N} \eta_{c}, \quad c \in C^{*} .
$$

Equivalently, we have

$$
B_{n}(C)=\prod_{c \in C^{*}} \exp \left(\lambda\left(\mathrm{e}^{2 \pi i n / N} \eta_{c}-\eta_{c}+\text { c.c. }\right)\right)
$$

This operator is the magnetic analog of the Wilson loop operator.

In the weakly-coupled gauge theory without dynamical monopoles, the 't Hooft loop operator creates a stable magnetic flux tube, and $\left\langle B_{n}(C)\right\rangle$ exhibits area-law decay. But when there are dynamical monopoles, $B_{n}(C)$ always exhibits perimeterlaw decay. The interpretation is clear. For any finite $\lambda$, a $\mathbb{Z}_{N}$ magnetic flux tube is unstable, for the tube can break via nucleation of a monopole-antimonopole pair.

Note that the operator defined by eq. (10.11) makes sense even if $C$ is an open path rather than a closed loop. That is, we may define in like fashion an operator

$$
B_{n}\left(P_{i, j}\right)=\prod_{c \in P_{i, j}^{*}} \exp \left(\lambda\left(\mathrm{e}^{2 \pi i n / N} \eta_{c}-\eta_{c}+\text { c.c. }\right)\right),
$$


where $i$ and $j$ are sites of the dual lattice, and $P_{i, j}$ is a path connecting these sites. This operator creates a monopole-antimonopole pair, connected by a Dirac string; it can be used to compute the mass of a dynamical monopole. Obviously, it is closely analogous to the operator eq. (10.8).

Having now in hand the operator $B_{n}(C)$ that introduces a classical $\mathbb{Z}_{N}$ monopole on the world-line $C$, and the operator $G^{(\nu)}(\Sigma)$ that introduces a classical $\mathbb{Z}_{N}$ electric flux tube on the world sheet $\Sigma$, we are ready to construct the operator, analogous to $A_{n}^{(\nu)}(\Sigma, C)$, that probes the Aharonov-Bohm interaction between monopoles and electric flux tubes; it is

$$
E_{n}^{(\nu)}(\Sigma, C)=\frac{G^{(\nu)}(\Sigma) B_{n}(C)}{\left\langle G^{(\nu)}(\Sigma)\right\rangle\left\langle B_{n}(C)\right\rangle}
$$

If there is an infinite-range Aharonov-Bohm interaction, the expectation value of this operator will have the asymptotic behavior

$$
\lim \left\langle E_{n}^{(\nu)}\right\rangle=\frac{1}{n_{\nu}} \chi^{(\nu)}\left(\left(\mathrm{e}^{2 \pi i n / N}\right)^{k(\Sigma, C)}\right),
$$

where $k(\Sigma, C)$ is the linking number of $\Sigma$ and $C$.

Finally, we remark that it is straightforward to generalize the construction in sect. 5, and define an operator that creates a loop of non-abelian cosmic string, in a theory that contains dynamical monopoles. We need only be cognizant of the change in the plaquette action that occurs when dynamical monopoles are included; a string that carries flux $a \in \mathrm{SU}(N)$ is now created by

$$
B_{a}\left(C, \Sigma, x_{0}\right)=\prod_{P \in \Sigma^{*}} \exp \left\{\beta\left(\operatorname{tr}\left(V_{l_{P}} a V_{l_{P}}^{-1} \eta_{P} U_{P}\right)-\operatorname{tr}\left(\eta_{P} U_{P}\right)\right)+\text { c.c. }\right\},
$$

where $\Sigma$ is a surface on the dual lattice, bounded by the loop $C$. When $C$ shrinks to a point, we obtain the operator $F_{a}\left(\Sigma, x_{0}\right)$ that introduces a classical string source on the closed world-sheet $\Sigma$.

\subsection{MONOPOLE CONDENSATION}

A pure $\mathrm{SU}(N)$ gauge theory, without dynamical monopoles, is confining at strong coupling. For $\beta \ll 1$, gauge field fluctuations are unsuppressed, and the resulting magnetic disorder gives rise to stable electric flux tubes. We want to explore how dynamical magnetic monopoles modify the physics of this theory.

First, we consider the parameter regime $\beta \ll 1$ and $\lambda \ll 1$, so that virtual monopoles are unsuppressed. It is easy to anticipate what will happen. A "monopole condensate" will form, which, in effect, will spontaneously break the local $\mathbb{Z}_{N}$ symmetry of the theory. Thus, the electric flux tube will become the boundary of a 
domain wall. As usual, this domain wall will decay by quantum tunneling - an electric flux tube will spontaneously nucleate, and expand, consuming the wall. Thus, there will be no stable electric flux tubes, and no infinite range AharonovBohm interaction between flux tubes and monopoles.

We can check whether this expectation is correct in strong-coupling perturbation theory. We proceed by expanding $\mathrm{e}^{-S_{\text {plaq }}}$ in powers of $\beta$ at each plaquette, and $\mathrm{e}^{-S_{\text {cube }}}$ in powers of $\lambda$ at each cube. Roughly speaking, the terms that survive when the $U_{l}$ and $\eta_{P}$ are summed are ones such that a set of "tiled" cubes forms a closed (three-dimensional) hypersurface, or else the tiled cubes form an open hypersurface that is bounded by a (two-dimensional) surface of tiled plaquettes. In other words, strong-coupling perturbation theory can be interpreted as a sum over histories for (heavily suppressed) domain walls bounded by electric flux tubes.

Consider, now, the behavior of $G^{(f)}(\Sigma)$, where $(f)$ denotes the defining representation of $\operatorname{SU}(N)$. If $\beta=0$, then the leading contribution to $\left\langle G^{(f)}(\Sigma)\right\rangle$, for $\lambda \ll 1$, is obtained by tiling the minimal hypersurface that is bounded by $\Sigma$; thus we have

$$
\left\langle G^{(f)}(\Sigma)\right\rangle \sim(\lambda)^{\text {Volume( } \Sigma)}
$$

The interpretation is that $G^{(f)}(\Sigma)$ is the boundary of a domain wall, where the wall tension is $\sigma \sim-\epsilon^{-3} \ln (1 / \lambda)$ ( $\epsilon$ is the lattice spacing). But for $\beta>0$, this domain wall is unstable. When the surface $\Sigma$ is very large, a much larger contribution to $\left\langle G^{(f)}(\Sigma)\right\rangle$ is obtained by tiling the plaquettes of $\Sigma$; this contribution is

$$
\left\langle G^{(f)}(\Sigma)\right\rangle \sim(\beta / N)^{\mathrm{Area}(\Sigma)} .
$$

(As $\beta \rightarrow 0$, the electric flux tube becomes arbitrarily heavy, and the domain wall is arbitrarily long-lived.)

Accordingly, the operator $W^{(f)}(C, \Sigma)$ does not create a stable electric flux tube. The leading behavior of its expectation value, too, is found by tiling the plaquettes of $\Sigma$, so that

$$
\left\langle W^{(f)}(C, \Sigma)\right\rangle \sim(\beta / N)^{\operatorname{Area}(\Sigma)}
$$

There is no dependence on the area of the minimal surface bounded by $C$ (compare eq. (7.2)), signifying that the dynamical flux tube created by $W^{(f)}(C, \Sigma)$ has vanishing tension.

It is obvious that the leading contribution to $\left\langle G^{(f)}(\Sigma)\right\rangle$ is unaffected by an insertion of $B_{n}(C)$, even if $C$ and $\Sigma$ link. So we have

$$
\lim \left\langle E_{n}^{(f)}(\Sigma, C)\right\rangle=1
$$

there is no long-range Aharonov-Bohm interaction. 
Incidentally, we could have chosen the cube action to be

$$
S_{c}=-\lambda\left(\eta_{c}\right)^{m}+\text { c.c.. }
$$

Then, in effect, for $\lambda \ll 1$, charge- $m$ monopoles condense; this breaks the local symmetry to $\mathbb{Z}_{M}$, where $M$ is the greatest common factor of $N$ and $m$ (compare sect. 4). In other words, $W^{(\nu)}(C, \Sigma)$ does create a stable flux tube, if a source transforming as the representation $(\nu)$ has no Aharonov-Bohm interaction with a charge- $m$ monopole (so that the tube is not the boundary of a wall), and if ( $\nu$ ) represents $\mathbb{Z}_{M}$ non-trivially (so that the tube does not break).

\subsection{MAGNETIC HAIR}

Now we consider the regime $\beta \ll 1$ and $\lambda \gg 1$. In this limit, virtual monopoles are heavily suppressed. We anticipate that quantum-mechanical magnetic charge fluctuations will not wipe out the infinite-range Aharonov-Bohm interaction between monopoles and electric flux tubes.

Again, we can check this expectation against perturbation theory. Weak-coupling perturbation theory, for $\lambda \gg 1$ is an expansion in the number of frustrated cubes. The frustrated cubes form closed loops that we may interpret as the world-lines of virtual monopoles. The expansion in $\beta$, as before, is an expansion in the number of tiled plaquettes. The tiled plaquettes form closed surfaces that we may interpret as the world-sheets of electric flux tubes. Perturbation theory, then, is a sum over histories for (heavily suppressed) magnetic monopoles and electric flux tubes.

For example, consider $\left\langle G^{(f)}(\Sigma)\right\rangle$. The leading non-trivial contribution arises from a configuration such that (in a particular gauge) $\eta_{P}=\mathrm{e}^{ \pm 2 \pi i / N}$ for a single plaquette $P$ contained in $\Sigma^{*}$, while $\eta_{P}=1$ for all other plaquettes. Flipping one plaquette frustrates four cubes, so this contribution gives

$$
\left\langle G^{(f)}(\Sigma)\right\rangle \sim 1+\sum_{n= \pm 1}\left(\mathrm{e}^{2 \pi i n / N}-1\right)(\exp [-2 \lambda(1-\cos (2 \pi / N))])^{4} .
$$

Summing disconnected contributions causes the result to exponentiate; we find

$$
\left\langle G^{(f)}(\Sigma)\right\rangle \sim \exp \left(-\kappa^{(\text {ren })} \operatorname{Area}(\Sigma)\right),
$$

where

$$
\epsilon^{2} \kappa^{(\text {ren })} \sim 2(1-\cos (2 \pi / N)) \exp (-8 \lambda[1-\cos (2 \pi / N)])
$$

(and $\epsilon$ is the lattice spacing). The interpretation is that, because of the Aharonov-Bohm interaction between monopoles and flux tubes, inserting $G^{(f)}(\Sigma)$ modifies the contribution to the vacuum energy due to virtual monopole pairs that wind around $\Sigma$, resulting in a renormalization of the tension of the classical string source. 
When we compute $\left\langle W^{(f)}(C, \Sigma)\right\rangle$, a similar renormalization of the tension of the classical string on $\Sigma$ occurs. But in addition, for $\beta \ll 1$, the configurations that contribute have a surface of tiled plaquettes bounded by $C$. The leading behavior, then, is

$$
\left\langle W^{(f)}(C, \Sigma)\right\rangle \sim(\beta / N)^{\operatorname{Area}(C)} \exp \left(-\kappa^{(\text {ren })} \operatorname{Area}(\Sigma)\right) .
$$

We conclude that $W^{(f)}(C, \Sigma)$ creates a stable electric flux tube with string tension

$$
\epsilon^{2} \kappa^{(\mathrm{dyn})} \sim-\ln (\beta / N) .
$$

When the operator $B_{n}(C)$ is inserted, it tends to frustrate the cubes in $C^{*}$. But, as we already noted in subsection 10.3, frustrated cubes can be avoided if the $\eta_{P}$ assume a suitable configuration. We may choose an arbitrary surface $T$ on the dual lattice whose boundary is $C$. Dual to the plaquettes of $T$ is a set of plaquettes $T^{*}$ of the original lattice. The desired configuration (in a particular gauge) is

$$
\begin{array}{ll}
\eta_{P}=\mathrm{e}^{2 \pi i n / N}, & P \in T^{*}, \\
\eta_{P}=1, & P \notin T^{*} .
\end{array}
$$

(The local symmetry transformation eq. (10.3) deforms the surface $T$, but leaves its boundary intact.)

By summing over gauge field fluctuations about the configuration eq. (10.26), we find the leading behavior

$$
\left\langle B_{n}(C)\right\rangle \sim \exp \left(-M_{n}^{(\text {ren })} \operatorname{Perimeter}(C)\right),
$$

where

$$
\epsilon M_{n}^{(\mathrm{ren})} \sim 2 N^{2}(\beta / N)^{6}(1-\cos (2 \pi n / N))
$$

is the renormalization of the mass of the classical monopole source. This renormalization is associated with virtual electric flux tubes whose world-sheets link the world line of the monopole, and arises because of the Aharonov-Bohm interaction between monopole and flux tube.

When the operator $B_{n}\left(P_{i, j}\right)$ is inserted, a line of frustrated cubes connecting $i$ and $j$ cannot be avoided, and so we find the leading behavior

$$
\left\langle B_{n}\left(P_{i, j}\right)\right\rangle \sim \exp \left(-M_{n}^{(\mathrm{dyn})} \operatorname{Distance}(i, j)\right) \exp \left(-M_{n}^{\text {(ren) }} \operatorname{Length}(P)\right),
$$

where

$$
\epsilon M_{n}^{(\mathrm{dyn})} \sim 2 \lambda(1-\cos (2 \pi n / N))
$$




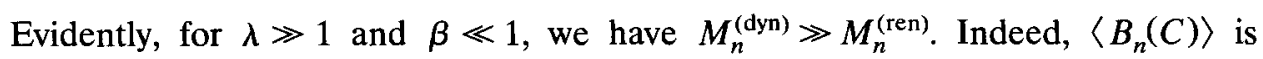
dominated by small fluctuations about the configuration eq. (10.26) for precisely this reason - the renormalization of the classical source is much less costly than screening the source with dynamical monopoles.

With the $\eta_{P}$ in the configuration eq. (10.26), the operator $G^{(f)}(\Sigma)$ assumes the value $\exp (2 \pi i n k / N)$, where $k$ is the linking number of $\Sigma$ and $C$. Furthermore, except on the loop $C$, this configuration is locally equivalent to the trivial configuration with $\eta_{P}=1$ everywhere. Thus, as we expand in the small fluctuations about eq. (10.26), we find, to each order of the expansion,

$$
\lim \left\langle E_{n}^{(f)}\right\rangle=\left(\mathrm{e}^{2 \pi i n / N}\right)^{k(\Sigma, C)}
$$

We see that, at least to each order of perturbation theory, our expectation is confirmed. In a confining theory that contains weakly coupled dynamical magnetic monopoles, there is an infinite-range Aharonov-Bohm interaction between monopoles and electric flux tubes.

We thank Hoi-Kwong Lo, and, especially, Martin Bucher, for helpful discussions. This work was supported by DOE grant DE-AC03-81-ER40050, and by NSF grants NSF-PHY-87-14654, NSF-PHY-90-21984 and NSF-PHY-89-04035. J.M.R. and M.G.A. wish to thank the Aspen Center for Physics and, especially, the Caltech Physics Department, for their hospitality during portions of this work.

\section{Appendix A}

In this appendix we consider in more detail some of the lattice perturbation theory calculations mentioned in the body of the text. Specifically, we calculate, in a pure gauge theory, the behavior of $\left\langle F_{a}\left(\Sigma, x_{0}\right)\right\rangle$ and $\left\langle B_{a}\left(\Sigma, C, x_{0}\right)\right\rangle$ in leading order in both strong and weak coupling perturbation theory. We demonstrate that $\left\langle F_{a}\left(\Sigma, x_{0}\right)\right\rangle$ exhibits an area law decay in both limits, and that only in the weak coupling limit does $\left\langle B_{a}\left(\Sigma, C, x_{0}\right)\right\rangle$ create a dynamical string on $C$. We also consider the problem that arises when one attempts to use the untraced Wilson loop operator eq. (5.15) to construct an Aharonov-Bohm order parameter.

\section{A.1. PURE GAUGE THEORY: STRONG COUPLING}

We start with the calculation of $\left\langle F_{a}\left(\Sigma, x_{0}\right)\right\rangle$ and $\left\langle B_{a}\left(\Sigma, C, x_{0}\right)\right\rangle$ in the strongcoupling regime $(\beta \ll 1)$ of the pure gauge theory defined by the plaquette action eq. (5.11), namely

$$
S_{\mathrm{gauge}, P}^{(R)}=-\beta \chi^{(R)}\left(U_{P}\right)+\text { c.c.. }
$$


For definiteness we will assume that the representation $(R)$ that defines the theory is irreducible, and that it satisfies the constraints that $R \otimes R$ does not contain the trivial representation, while $R \otimes R^{*}$ contains it exactly once.

The expectation value $\left\langle F_{a}\left(\Sigma, x_{0}\right)\right\rangle$ is given by

$$
\left\langle F_{a}\left(\Sigma, x_{0}\right)\right\rangle=\frac{\left\langle\left\langle F_{a}\left(\Sigma, x_{0}\right)\right\rangle\right\rangle}{\langle\langle 1\rangle\rangle},
$$

where the unnormalized expectation value of an operator $X$ is defined by

$$
\langle\langle X\rangle\rangle=\prod_{\langle i j\rangle} \int \mathrm{d} U_{\langle i j\rangle}\left(X \prod_{P} \exp \left(-S_{P}\right)\right)
$$

To find the renormalized string tension we need to calculate the leading behavior of both $\left\langle\left\langle F_{a}\left(\Sigma, x_{0}\right)\right\rangle\right\rangle$ and $\langle\langle 1\rangle\rangle$. The perturbation expansion in the strong-coupling regime is of the form of a sum over closed surfaces, in which surfaces of greater area are suppressed by powers of $\beta$ compared to smaller ones. Formally, the strong-coupling expansion proceeds by performing a character expansion on the exponentiated Wilson action,

$$
\exp \left(\beta\left(\chi^{(R)}\left(U_{P}\right)+\chi^{\left(R^{*}\right)}\left(U_{P}\right)\right)\right)=N(\beta)\left(1+\sum_{(\nu) \neq 1} C^{(\nu)}(\beta) \chi^{(\nu)}\left(U_{P}\right)\right) .
$$

The link integrations then select out closed surfaces $S$, each formed by "tiling" $S$ with factors of $\chi^{(\nu)}\left(U_{P}\right)$ for each plaquette $P \in S$. The leading non-trivial contribution will be from the smallest surfaces, which furthermore are tiled with characters whose associated factors of $C^{(\nu)}(\beta)$ have the lowest non-trivial dependence on $\beta$. The factors $C^{(\nu)}(\beta)$ are found by multiplying eq. (A.4) by $\chi^{\left(\nu^{*}\right)}\left(U_{P}\right)$, summing $U_{P}$ over the group, and using the character orthogonality relations along with the assumed properties of the representation $(R)$, giving

$$
C^{(R)}(\beta)=1+\beta+\mathrm{O}\left(\beta^{2}\right), \quad C^{\left(R^{*}\right)}(\beta)=1+\beta+\mathrm{O}\left(\beta^{2}\right),
$$

with all other irreducible representations acquiring their first non-trivial $\beta$-dependence at $O\left(\beta^{2}\right)$. Therefore the leading contribution will come from surfaces tiled with characters in the representations $(R)$ and $\left(R^{*}\right)$.

In the evaluation of $\left\langle F_{a}\right\rangle$ we must, in addition, identify the leading contribution that is sensitive to the presence of $F_{a}$, and hence does not cancel between $\left\langle\left\langle F_{a}\right\rangle\right\rangle$ and $\langle\langle 1\rangle\rangle$. All closed surfaces that do not intersect $\Sigma$ are unaffected by the presence of $F_{a}$. Therefore we factorize both $\left\langle\left\langle F_{a}\right\rangle\right\rangle$ and $\langle\langle 1\rangle\rangle$ in eq. (A.2) into contributions from surfaces "on- $\Sigma$ " and "off- $\Sigma$ ", the off- $\Sigma$ dependence cancelling between them. The dominant on- $\Sigma$ contribution is shown in fig. A.1, where all six 


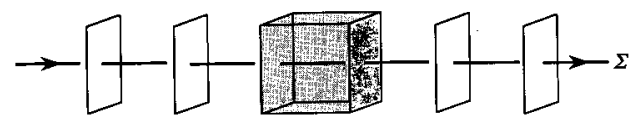

Fig. A.1. The leading contribution to $\left\langle F_{a}\left(\Sigma, x_{0}\right)\right\rangle$, in the strong-coupling limit of the pure gauge theory, arises from tiling a cube (shaded) that contains two plaquettes of $\Sigma^{*}$.

faces are tiled with factors of either $\chi^{(R)}$, or $\chi^{\left(R^{*}\right)}$. This results in a contribution to $\left\langle\left\langle F_{a}\right\rangle\right\rangle$ from fig. A.1 of

$$
2\left(\beta / n_{R}\right)^{6}\left|\chi^{(R)}(a)\right|^{2}
$$

where $n_{R}$ is the dimension of the representation $(R)$. The factor of $\left(n_{R}\right)^{-6}$ comes from the six non-trivial link integrations, and the factor of 2 from the two equal contributions of either $\chi^{(R)}$ or $\chi^{\left(R^{*}\right)}$. Since the number of possible positions of this elementary cube on the surface $\Sigma$ is given by the area $A(\Sigma)$ of $\Sigma$ (measured in terms of the size of the elementary plaquette $\epsilon^{2}$ ), to get the contribution of a single elementary cube located anywhere on $\Sigma$ we should further multiply (A.6) by $A(\Sigma)$.

The area law decay of $\left\langle F_{a}\right\rangle$ arises from considering an arbitrary number of such elementary tiled cubes on the surface $\Sigma$, rather than just a single contribution. In the "dilute gas" approximation where we can ignore correlations between the various elementary cubes, this leads to an exponentiation of the above result with a overall factor of $A(\Sigma)$. Finally, the "on- $\Sigma$ " contributions to $\langle\langle 1\rangle\rangle$ lead to a similar exponential dependence on $A(\Sigma)$, except that the group element $a$ is replaced by the identity element. Putting these together yields the result

$$
\left\langle F_{a}\left(\Sigma, x_{0}\right)\right\rangle=\exp \left(2\left(\beta / n_{R}\right)^{6}\left(\left|\chi^{(R)}(a)\right|^{2}-n_{R}^{2}\right) A(\Sigma)\right)
$$

In other words, the renormalized classical string tension defined in eq. (6.4) is given by

$$
\epsilon^{2} \kappa_{a}^{\text {(ren })} \sim 2\left(\frac{\beta}{n_{R}}\right)^{6}\left(n_{R}^{2}-\left|\chi^{(R)}(a)\right|^{2}\right)
$$

to lowest order in $\beta$.

The calculation of $\left\langle B_{a}\left(\Sigma, C, x_{0}\right)\right\rangle$ proceeds in a very similar way; the only question concerns the behavior at the boundary curve $C$. Here there exist contributions to $\left\langle B_{a}\left(\Sigma, C, x_{0}\right)\right\rangle$ similar to those of fig. A.1 except only one plaquette $P \in \Sigma^{*}$ is contained in the cube. This leads to an additional decay of $\left\langle B_{a}\left(\Sigma, C, x_{0}\right)\right\rangle$ depending on the length $P(C)$ of the perimeter [eq. (7.3)],

$$
\left\langle B_{a}\left(\Sigma, C, x_{0}\right)\right\rangle \sim \exp \left(-\kappa_{a}^{(\mathrm{ren})} A(\Sigma)\right) \exp \left(-m_{a}^{(\mathrm{ren})} P(C)\right),
$$


with

$$
\epsilon m_{a}^{(\text {ren })} \sim 2\left(\frac{\beta}{n_{R}}\right)^{6}\left(n_{R}^{2}-n_{R} \operatorname{Re} \chi^{(R)}(a)\right)
$$

Since the configurations that dominate the expectation values of $F_{a}$ and $B_{a}$ do not extend deep into the volume enclosed by $\Sigma$, we find that there are no stable dynamical strings (or vortices) in this regime, and therefore that there is no long-distance Aharonov-Bohm interaction between flux tubes and charges.

\section{A.2. WEAK COUPLING}

This is the regime in which we expect to find $A_{a}^{(\nu)}\left(\Sigma, x_{0} ; C\right)$ displaying a non-trivial Aharonov-Bohm interaction. To start we need to discover which configurations dominate the behavior of $\left\langle F_{a}\left(\Sigma, x_{0}\right)\right\rangle$. When $\beta \gg 1$, frustrated plaquettes (ones with non-minimal gauge action) are highly suppressed. However, as described in subsect. 6.2, an insertion of $F_{a}\left(\Sigma, x_{0}\right)$ tends to frustrate the plaquettes in $\Sigma^{*}$, unless the link variables assume a suitable configuration. The configuration that leaves no frustrated plaquettes, and thus acts as the "ground state" in the presence of $F_{a}$ is illustrated in fig. 9. It consists of a "forest" of links inside $\Sigma$ that have value $U_{l}=a$. Specifically we choose a three-dimensional hypersurface $\Omega$ made up of cubes of the dual lattice that has boundary $\Sigma$. Dual to these cubes is a set of links $\Omega^{*}$ on the original lattice. The configuration with no excited plaquettes is then given by eq. (6.7), namely $U_{l}=a$ for $l \in \Omega^{*}$, and $U_{l}=e$ otherwise. The area-law decay of $\left\langle F_{a}\left(\Sigma, x_{0}\right)\right\rangle$ in the weak coupling regime arises from fluctuations of the link variables around this state, which are sensitive to the presence of the non-trivial background.

The leading such contribution to the decay of $\left\langle F_{a}\left(\Sigma, x_{0}\right)\right\rangle$ in the pure gauge theory is shown in fig. A.2a. (For simplicity we discuss the situation for vortices in three euclidean dimensions.) It consists of a configuration that has nine excited plaquettes, and is constructed by considering two neighboring plaquettes in $\Sigma^{*}$. These plaquettes are connected with four links with $U_{l}=b$ to make a cube, with $b$ summed over the group. In this configuration, there are eight excited plaquettes with plaquette action proportional to $\beta \operatorname{Re} \chi(b)$ (the unshaded plaquettes in fig. A.2b). But, in addition, there is an excited plaquette (if $a$ and $b$ do not commute) with action proportional to $\beta \operatorname{Re} \chi\left(a b a^{-1} b^{-1}\right)$ (the shaded plaquette in fig. A.2b). This extra excitation distinguishes the contributions of fig. A.2 to $\left\langle\left\langle F_{a}\left(\Sigma, x_{0}\right)\right\rangle\right\rangle$ and $\langle\langle 1\rangle\rangle$. Changes of variable can move the ninth excited plaquette around, but cannot get rid of it.

Of course, the process illustrated in fig. A. 2 is only one of a set of similar processes where instead of summing over the same value $b$ for each of the four connecting links, we sum independently over their values $b_{i}$. These differ from the 


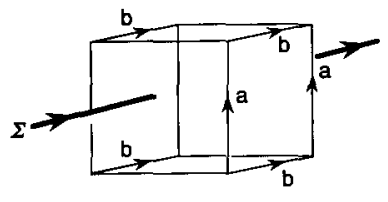

(a)
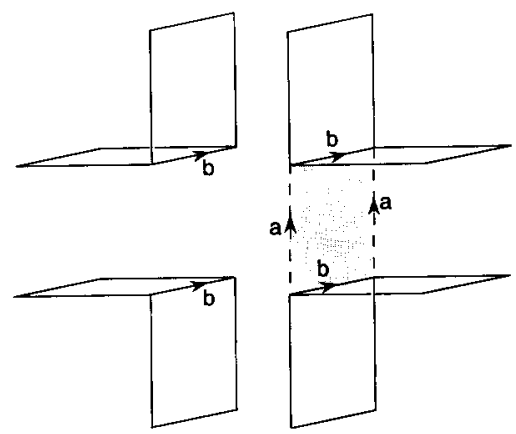

(b)

Fig. A.2. The leading contribution to $\left\langle F_{a}\left(\Sigma, x_{0}\right)\right\rangle$, in the weak-coupling limit of the pure gauge theory. The cube shown contains two plaquettes that are in $\Sigma^{*}$, and the link variables assume the indicated values. (Unmarked links have the value $U_{l}=1$.) There are altogether nine excited plaquettes - eight (unshaded) plaquettes with $U_{P}=b$ and one (shaded) plaquette with $U_{P}=a b a^{-1} b^{-1}$.

contribution of fig. A.2 in two ways: there will either be a greater number of excited plaquettes leading to a subdominant contribution, or, if some of the $b_{i}$ are taken to be the identity, changes of variable on the remaining, independent, $b_{i}$ show that the $a$-links have no physical effect. This is why, for instance, we do not consider the less suppressed, and apparently non-trivial, contribution arising from the excitation of just two joining links.

We can understand this in physical terms. The contribution to $\left\langle F_{a}\left(\Sigma, x_{0}\right)\right\rangle$ reflects how the non-trivial boundary condition introduced by the vortex (or string) affects the quantum fluctuations of the gauge fields. To see the effect, we must consider processes in which virtual gauge field excitations (with gauge quantum numbers that Aharonov-Bohm scatter off the inserted string of flux $a$ ) propagate around the string. The process illustrated in fig. A.2 is the leading one of this type.

The end result of these considerations is a renormalization of the classical string tension (or vortex mass in three dimensions) leading to an area-law (respectively, perimeter-law) decay of $\left\langle F_{a}\left(\Sigma, x_{0}\right)\right\rangle$ as in eq. (6.4). An estimate of the renormalized vortex mass in the weak-coupling regime arising from fig. A.2 is

$$
\begin{aligned}
\epsilon m_{a}^{(\mathrm{ren})} \sim & \frac{1}{n_{\mathrm{G}}} \sum_{b \neq 1}\left(\exp \left\{(-16 \beta)\left[n_{R}-\operatorname{Re} \chi^{(R)}(b)\right]\right\}\right. \\
& \left.\times\left(1-\exp \left\{(-2 \beta)\left[n_{R}-\operatorname{Re} \chi^{(R)}\left(b a b^{-1} a^{-1}\right)\right]\right\}\right)\right),
\end{aligned}
$$

where we require more information about the group $G$ to explicitly evaluate the sum. 
Also, note that we have found area law decay of $\left\langle F_{a}\left(\Sigma, x_{0}\right)\right\rangle$ in both regimes of the pure non-abelian gauge theory described by eq. (A.1) (when $a$ is not in the center of $G$ ). This differs from the situation in a pure abelian gauge theory where a change of variables shows that $\left\langle F_{a}\left(\Sigma, x_{0}\right)\right\rangle \equiv 1$ [5]. This is to be expected since the pure non-abelian gauge theory contains excitations that, via the Aharonov-Bohm effect, are sensitive to the introduction of flux on $\Sigma$.

To see that there exist stable dynamical strings in this regime of the pure gauge theory, we must consider $\left\langle B_{a}\left(\Sigma, C, x_{0}\right)\right\rangle$, rather than $\left\langle F_{a}\left(\Sigma, x_{0}\right)\right\rangle$. Since the surface $\Sigma$ now has a boundary, the three-dimensional hypersurface $\Omega$ must also end on a two-dimensional surface $S$. This surface is dual to a set of plaquettes $S^{*}$ on the original lattice that must be frustrated, since only one of their links is contained within $\Omega^{*}$. Clearly the dominant configuration is the one in which $\mathrm{S}$ is the minimal area surface with boundary $C$. Each such plaquette is suppressed relative to the corresponding vacuum configuration that dominates $\langle\langle 1\rangle\rangle$ by an amount

$$
\exp \left(2 \beta\left(\operatorname{Re} \chi^{(R)}(a)-n_{R}\right)\right)
$$

In other words $\left\langle B_{a}\left(\Sigma, C, x_{0}\right)\right\rangle$ behaves as in eq. (7.2)

$$
\left\langle B_{a}\left(\Sigma, C, x_{0}\right)\right\rangle=\exp \left(-\kappa_{a}^{(\mathrm{ren})} A(\Sigma)\right) \exp \left(-\kappa_{a}^{(\mathrm{dyn})} A(C)\right)
$$

where $A(C)$ is the area of the minimal surface $S$, and $\kappa_{a}^{(\mathrm{dyn})}$ is the dynamical string tension of the stable string with flux in the a conjugacy class,

$$
\epsilon^{2} \kappa_{a}^{(\mathrm{dyn})} \sim 2 \beta\left(n_{R}-\operatorname{Re} \chi^{(R)}(a)\right) .
$$

In some situations it is possible that $B_{a}\left(\Sigma, C, x_{0}\right)$ creates not just one dynamical string, of flux $a$, on $C$, but instead creates a number of strings of varying fluxes $a_{1}, \ldots, a_{k}$, where $a_{1} a_{2} \ldots a_{k}=a$. The corresponding link configuration would be a "forest state" of links of value $U_{l}=a$ that terminates gradually in a number of steps rather than dropping to the identity across a single plaquette. This is the more favorable configuration if the total area law suppression of the multiple strings is less than that for the single string. This translates into the condition (in the pure gauge case, and ignoring perimeter corrections that vanish in the infinite size limit)

$$
(k-1) n_{R}+\operatorname{Re} \chi^{(R)}(a)-\sum_{i=1}^{k} \operatorname{Re} \chi^{(R)}\left(a_{i}\right)<0 .
$$

As an example of this, consider the expectation value, in a pure $\mathbb{Z}_{6}$ gauge theory, of the operator $B_{3}\left(\Sigma, C, x_{0}\right)$ that attempts to create a dynamical string with flux $a=\omega^{3}$ on $C$ (where $\left.\omega=\exp (2 \pi i / 6)\right)$. The dominant process in this case is not the 
creation of an $\omega^{3}$ string, but instead that of three $\omega$-strings, since, substituting into eq. (A.15), we find $2+(-1)-3(1 / 2)<0$.

We now turn to the order parameter $A_{a}^{(\nu)}\left(\Sigma, x_{0} ; C\right)$, which is defined (eq. (5.3)) in terms of $F_{a}(\Sigma)$ and the Wilson loop operator $W^{(\nu)}(C) . F_{a}(\Sigma)$ has been described above, so we will now discuss the Wilson loop operator, and demonstrate eq. (5.4).

First, let us consider the untraced Wilson operator $U^{(\nu)}\left(C, x_{0}\right)$ [eq. (5.15)]. It is easy to calculate the leading behavior of the expectation value of $U^{(\nu)}\left(C, x_{0}\right)$ at weak coupling: as above we factorize the contributions from links on- $C$ and off- $C$, and cancel the off- $C$ contributions,

$$
\frac{\left\langle\left\langle U^{(\nu)}\left(C, x_{0}\right)\right\rangle\right\rangle}{\langle\langle 1\rangle\rangle}=\frac{\sum_{U_{1}} \ldots \sum_{U_{L}} D^{(\nu)}\left(U_{1} \ldots U_{L}\right) \prod_{i=1}^{L} \exp \left(4 D \beta \operatorname{Re} \chi^{(R)}\left(U_{i}\right)\right)}{\sum_{U_{1}} \cdots \sum_{U_{L}} \prod_{i=1}^{L} \exp \left(4 D \beta \operatorname{Re} \chi^{(R)}\left(U_{i}\right)\right)},
$$

where we work in $D+1$ euclidean dimensions, and $U_{i}$ for $i=1, \ldots, L$ are the links in $C$. (In eq. (A.16), we sum over all values of the link variables contained in $C$, but keep all other link variables fixed at $U_{l}=1$.) Because we have not traced the Wilson loop the multiple summations factorize. We then use

$$
\frac{1}{n_{\mathrm{G}}} \sum_{g \in \mathrm{G}} D^{\left(\nu^{*}\right)}(g) \chi^{(\mu)}(g)=\frac{1}{n_{\nu}} \delta^{\mu, \nu} I^{(\nu)},
$$

where $I^{(\nu)}$ is the identity matrix. Along with eq. (A.4) this implies that

$$
\left\langle U^{(\nu)}\left(C, x_{0}\right)\right\rangle=\left(\frac{C^{\left(\nu^{*}\right)}(2 D \beta)}{n_{\nu}}\right)^{L} I^{(\nu)} .
$$

If we repeat the calculation in the background created by a string insertion operator $F_{a}\left(\Sigma, x_{0}\right)$ then the only difference is that for each time $C$ links $\Sigma$ there is a link in $C$ that is multiplied by $a$. Neither $F_{a}\left(\Sigma, x_{0}\right)$ nor $U^{(\nu)}\left(C, x_{0}\right)$ is gauge-invariant, but a gauge transformation conjugates both $a$ and $U^{(\nu)}$ by the value of the transformation at $x_{0}$, so these conjugations cancel, and the gauge-invariant result is that $I^{(\nu)}$ is replaced by $D^{(\nu)}\left(a^{k(\Sigma, C)}\right)$, where $k(\Sigma, C)$ is the linking number of the two curves. This proves eq. (5.16), and by taking a trace it proves eq. (5.4).

However there is a major caveat that limits the usefulness of the untraced Wilson loop: there are corrections to eq. (5.16) that do not go to zero as $C$ and $\Sigma$ become large and well separated. Recall that $F_{a}\left(\Sigma, x_{0}\right)$ corresponds to a thought experiment in which a specific group element $a$ (not just a conjugacy class) was associated with a string, using an arbitrary basis at a point $x_{0}$. As described in subsect. 5.2 this means that in defining $F_{a}\left(\Sigma, x_{0}\right)$ we give each plaquette in $\Sigma^{*}$ a 
long "tail" of links that stretches back to $x_{0}$. The problem manifests itself (in a particular gauge) when we take into account configurations in which some (but not all) of the links coming out of $x_{0}$ are excited. In these configurations the inserted flux may be conjugated, but not the measured group element (or vice versa).

Concretely, the leading contribution, in weak coupling perturbation theory, that causes problems for the untraced Wilson loop is simply the excitation of a single link $u_{l}=b$ on the path that connects $x_{0}$ to $\Sigma$. In the three-dimensional case this leads to the excitation of four plaquettes, and is thus suppressed relative to the ground state configuration by a factor of $\exp \left(8 \beta\left(\operatorname{Re} \chi^{(R)}(b)-n_{R}\right)\right)$. However, the action of these configurations, though large at weak coupling, is completely independent of the size and separation of $\Sigma$ and $C$, and so remains finite as $\Sigma$ and $C$ become large and far apart. Their effect on the untraced order parameter is to conjugate the inserted flux (and thus the flux measured by $U^{(\nu)}\left(C, x_{0}\right)$ ). Therefore, there are corrections to eq. (5.16) that occur at finite order in weak-coupling perturbation theory that render the measured flux uncertain up to conjugation.

The physical interpretation of these configurations is that the path connecting $x_{0}$ to $\Sigma$ is linked by a virtual vortex-antivortex pair of flux $b$. In four dimensions there exist analogous processes where a virtual cosmic string links the connecting path. These effects do not correct the leading behavior of the ABOP given by eq. (5.4), because the traced Wilson loop is gauge-invariant, and so is not sensitive to conjugation of the inserted flux.

\section{References}

[1] J. Preskill, Vortices and monopoles, in Architecture of the fundamental interactions at short distances, ed. P. Ramond and R. Stora (North-Holland, Amsterdam, 1987)

[2] Y. Aharonov and D. Bohm, Phys. Rev. 119 (1959) 485

[3] R. Rohm, Princeton University Ph.D. thesis (unpublished) (1985); M.G. Alford and F. Wilczek, Phys. Rev. Lett. 62 (1989) 1071

[4] L. Krauss and F. Wilczek, Phys. Rev. Lett. 62 (1989) 1221

[5] J. Preskill and L. Krauss, Nucl. Phys. B341 (1990) 50

[6] M.G. Alford, J. March-Russell and F. Wilczek, Nucl. Phys. B337 (1990) 695

[7] M.J. Bowick et al., Phys. Rev. Lett. 61 (1988) 2823

[8] J. Preskill, Phys. Scr. T36 (1991) 258

[9] S. Coleman, J. Preskill and F. Wilczek, Mod. Phys. Lett. A6 (1991) 1631; Phys. Rev. Lett. 67 (1991) 1975; Nucl. Phys. B378 (1992) 175

[10] F. Bais, Nucl. Phys. B170[FS1] (1980) 32;

T.W.B. Kibble, Phys. Rep. 67 (1980) 183

[11] A.S. Schwarz, Nucl. Phys. B208 (1982) 141

[12] S. Coleman and P. Ginsparg, (1983), unpublished

[13] M. Alford, et al., Phys. Rev. Lett. 64 (1990) 1632 [Erratum: 65 (1990) 668]; Nucl. Phys. B349 (1991) 414

[14] M.G. Alford and J. March-Russell, Nucl. Phys. B 369 (1992) 276

[15] K. Wilson, Phys. Rev. D10 (1974) 2445

[16] G. 't Hooft, Nucl. Phys. B138 (1978) 1

[17] K. Fredenhagen and M. Marcu, Commun. Math. Phys. 92 (1983) 81 
[18] K. Fredenhagen, Particle structure of gauge theories, in Fundamental problems of gauge field theory, ed. G. Velo and A.S. Wightman (Plenum, New York, 1986)

[19] E. Fradkin and S. Shenker, Phys. Rev. D19 (1979) 3682;

T. Banks and E. Rabinovici, Nucl. Phys. B160 (1979) 349;

S. Dimopoulos, S. Raby and L. Susskind, Nucl. Phys. B173 (1980) 208

[20] K. Fredenhagen and M. Marcu, Phys. Rev. Lett. 56 (1986) 223

[21] M. Alford, S. Coleman and J. March-Russell, Nucl. Phys. B351 (1991) 735

[22] S. Coleman, The magnetic monopole fifty years later, in The unity of the fundamental interactions, ed. A. Zichichi (Plenum, New York, 1983)

[23] M. Srednicki and L. Susskind, Nucl. Phys. B179 (1981) 239

[24] M. Bucher, Nucl. Phys. B350 (1991) 163

[25] F. Wilczek and Y.-S. Wu, Phys. Rev. Lett. 65 (1990) 13

[26] J. Kiskis, Phys. Rev. D17 (1978) 3196

[27] T. Banks, Nucl. Phys. B323 (1989) 90

[28] K. Li, Nucl. Phys. B361 (1991) 437

[29] M.G. Alford and J. March-Russell, Discrete gauge theories, Princeton preprint PUPT-91-1234 (1991), to be published in Int. J. Mod. Phys. B

[30] M. Bucher, K.-M. Lee and J. Preskill, On detecting discrete Cheshire charge, CALTECH preprint CALT-68-1753 (1991), to be published in Nucl. Phys. B

[31] V. Poénaru and G. Toulouse, J. Phys. (Paris) 38 (1977) 887;

N.D. Mermin, Rev. Mod. Phys. 51 (1979) 591

[32] T.W.B. Kibble, G. Lazarides and Q. Shafi, Phys. Rev. D26 (1982) 435

[33] G. Mack and V. Petkova, Ann. Phys. (N.Y.) 123 (1979) 442

[34] A. Ukawa, P. Windey and A. Guth, Phys. Rev. D21 (1980) 1013 